\title{
European Society of Hypertension recommendations for conventional, ambulatory and home blood pressure measurement
}

\author{
Eoin O'Brien, Roland Asmar, Lawrie Beilin, Yutaka Imai, Jean-Michel Mallion, \\ Giuseppe Mancia, Thomas Mengden, Martin Myers, Paul Padfield, \\ Paolo Palatini, Gianfranco Parati, Thomas Pickering, Josep Redon, \\ Jan Staessen, George Stergiou and Paolo Verdecchia, on behalf of the European \\ Society of Hypertension Working Group on Blood Pressure Monitoring
}

Journal of Hypertension 2003, 21:821-848

Keywords: blood pressure measurement, conventional blood pressure measurement, ambulatory blood pressure measurement, home blood pressure measurement, sphygmomanometers, European Society of Hypertension recommendations

Please refer to the Appendix for afilations.
Correspondence and requests for reprints to Professor Eoin O'Brien, Blood Pressure Unit, Beaumont Hospital, Dublin 9, Ireland.

E-mail: eobrien@iol.ie

Received 31 January 2003 Accepted 3 February 2003

\section{Introduction}

Over the past 20 years or so, the accuracy of the conventional Riva-Rocci/Korotkoff technique of blood pressure measurement has been questioned and efforts have been made to improve the technique with automated devices. In the same period, recognition of the phenomenon of white-coat hypertension, whereby some individuals with an apparent increase in blood pressure have normal, or reduced, blood pressures when measurement is repeated away from the medical environment, has focused attention on methods of measurement that provide profiles of blood pressure behaviour rather than relying on isolated measurements under circumstances that may in themselves influence the level of blood pressure recorded. These methodologies have included repeated measurements of blood pressure using the traditional technique, self-measurement of blood pressure in the home or work place, and ambulatory blood pressure measurement using innovative automated devices.

Blood pressure measurement is the basis for the diagnosis, management, treatment, epidemiology and research of hypertension, and the decisions affecting these aspects of hypertension will be influenced for better or worse by the accuracy of the measurement. An accurate blood pressure reading is a prerequisite, therefore, regardless of which technique is used, yet all too often the accuracy of measurement is taken for granted or ignored.

The purpose of this monograph is to serve as a reference source for other guidelines relating to hyper- tension and cardiovascular disease. The content is divided into four parts: Part $\mathrm{I}$ is devoted to issues common to all techniques of measurement, Part II to conventional blood pressure measurement (CBPM), Part III to ambulatory blood pressure measurement (ABPM) and Part IV to self blood pressure measurement (SBPM).

\section{Part I. Aspects of blood pressure measurement common to all techniques Factors affecting the technique Selecting an accurate device}

An accurate device is fundamental to all blood pressure measurement [1]; if the device used to measure blood pressure is inaccurate, attention to methodological detail is of little relevance. It is acknowledged that the accuracy of blood pressure measuring devices should not be based solely on claims from manufacturers, which can at times be somewhat extravagant. Instead, independent validation, with the results published in peer-reviewed journals, should be demanded [2]. However, manufacturers often ignore this recommendation and potential purchasers are generally unaware of this requirement, assuming - not unreasonably - that if a product reaches the market place, it will measure blood pressure accurately. Aware of this problem, the Association for the Advancement of Medical Instrumentation (AAMI) published standards for electronic and aneroid sphygmomanometers in 1987, which included a protocol for the evaluation of the accuracy of devices [3], and this was followed in 1990 by the protocol of the British Hypertension Society (BHS) [4]; both protocols were revised in 1993 [5,6]. These protocols, which 
differed in detail, had a common objective, namely the standardization of validation procedures to establish minimum standards of accuracy and performance, and to facilitate comparison of one device with another. A large number of blood pressure measuring devices have been evaluated according to one or both protocols [2]. On the basis of past experience, it is evident that the conditions demanded by the protocols are difficult to fulfil. As a result, the Working Group on Blood Pressure Monitoring of the European Society of Hypertension (ESH) has recently published a simplified protocol to facilitate the evaluation process, with the expectation that manufacturers will be more likely to submit their products for validation in order to obtain the minimum approval necessary for a device to be used in clinical practice. Ultimately, it is hoped that most, if not all devices on the market will be independently assessed for basic accuracy according to this protocol [7]. Blood pressure measuring devices have been assessed in the literature from time to time $[2,8,9]$, but as such surveys soon become outdated, no listings of validated devices are made in this monograph. This information may be obtained from websites such as those of the ESH or BHS [10,11].

European Union (EU) directives govern the safety and basic accuracy requirements of blood pressure measuring devices [12-14], and all member states are legally obliged to obtain EU certification, guaranteeing that a device has been subjected to quality assurance evaluation in conformity with these directives. Independent clinical evaluation of blood pressure measuring devices according to established international protocols $[5,6,15]$ is recommended by the EU directive, but it is not obligatory.

\section{Variability of blood pressure}

No matter which measurement device is used, blood pressure will always be a variable haemodynamic phenomenon that is influenced by many factors, not least being the circumstances of measurement itself. The observer should be aware of the considerable variability that may occur in blood pressure from moment to moment with respiration, emotion, exercise, meals, tobacco, alcohol, temperature, bladder distension and pain, and that blood pressure is also influenced by age, race and diurnal variation, usually being lowest during sleep [16-19]. If these influences, which are common to all circumstances of blood pressure measurement, are ignored or unrecognized, erroneous diagnosis and inappropriate management may result.

It is not always possible to modify all these factors, but we can minimize their effect by taking them into account in reaching a decision as to the relevance or otherwise of a particular blood pressure measurement [20-23]. Insofar as is practical, the patient should be relaxed in a quiet room at a comfortable temperature and a short period of rest should precede the measurement. When it is not possible to achieve optimum conditions, this should be noted with the blood pressure reading.

\section{White-coat hypertension}

Anxiety increases blood pressure, often by as much as $30 \mathrm{mmHg}$ or more, and excursions in blood pressure during the day can be as great as $50-60 \mathrm{mmHg}$ for systolic blood pressure [16-19]. This may be regarded as a physiological reaction - the 'fight and flight' phenomenon, often referred to as the 'defence', 'alarm' or 'alerting' reaction. It is commonly seen in the Accident and Emergency Departments of hospitals when patients are frightened and extremely anxious, but it may also occur in family doctor's offices and in the outpatient department. An alerting reaction to a doctor is commonly referred to as the "white-coat effect'. It may occur in both normotensive and hypertensive individuals [19].

This reaction must be distinguished from the phenomenon known as 'white-coat hypertension', a condition in which a normotensive individual becomes hypertensive during repeated clinic blood pressure measurement, but pressures then settle to normal outside the medical environment $[24,25]$. The importance of the phenomenon in clinical practice is that decisions to decrease blood pressure, and especially to administer drugs, should never be made on the basis of measurements taken in circumstances where the defence reaction is likely to be present. The degree of this reaction varies greatly from patient to patient, being absent in many, and it is not substantially influenced by reassurance and familiarization with the technique and circumstances of blood pressure measurement [18]. White-coat hypertension is best demonstrated by ambulatory blood pressure measurement, and will be considered in more depth in Parts III and IV.

\section{The procedure \\ Explanation to the individual}

The first step in blood pressure measurement is adequate explanation of the procedure, in an attempt to allay fear and anxiety, especially in nervous individuals. Those having blood pressure measured for the first time should be told that there is minor discomfort caused by inflation of the cuff.

\section{Patient education}

Many patients are anxious to learn more about high blood pressure, and some are anxious to learn how to measure their own blood pressure; illustrated instruction books are helpful in this context and details of this procedure will be considered in more depth in Part IV. 


\section{Attitude of observer}

Before taking the blood pressure, the observer should be in a comfortable and relaxed position, and should not rush the procedure, otherwise the cuff may be deflated too rapidly, resulting in underestimation of systolic and overestimation of diastolic pressures. If any interruption occurs, the exact measurement may be forgotten and an approximation made. The blood pressure should always be documented as soon as it has been measured.

\section{Attitude of patient}

Patients should be encouraged to relax and be advised that neither they nor the observer should talk for the few minutes before or during the blood pressure measurement.

\section{Posture of patient}

Posture affects blood pressure, with a general tendency for it to decrease from the lying to sitting or standing position. However, in most people posture is unlikely to lead to significant error in blood pressure measurement, provided the arm is supported at heart level. Nonetheless, it is advisable to standardize posture for individual patients, and in practice blood pressure is usually measured with the individual in the sitting position. Patients should be comfortable whatever their position. No information is available on the optimal time that an individual should remain in a particular position before a measurement, but $5 \mathrm{~min}$ is suggested for the lying and sitting positions and $1 \mathrm{~min}$ for standing. Some patients may exhibit postural hypotension, especially with certain antihypertensive drugs. When this is likely, blood pressure should be measured with the patient both lying and standing [20-23].

\section{Arm support}

If the arm in which measurement is being made is unsupported, as tends to happen when the individual is sitting or standing, isometric exercise is being performed, increasing blood pressure and heart rate. Diastolic blood pressure may be increased by as much as $10 \%$ by having the arm extended and unsupported during blood pressure measurement. The effect of isometric exercise is greater in hypertensive patients and in those taking $\beta$-blocking drugs [26,27]. It is essential, therefore, for the arm to be supported during blood pressure measurement, especially when the individual is in the standing position, and this is best achieved in practice by having the observer hold the person's arm at the elbow, although in research the use of an arm support on a stand has much to commend it [20-23].

\section{Arm position}

The forearm must also be at the level of the heart as denoted by the mid-sternal level. Dependency of the arm below heart level leads to an overestimation of systolic and diastolic pressures and raising the arm above heart level leads to underestimation. The magnitude of this error can be as great as $10 \mathrm{mmHg}$ for systolic and diastolic readings. This source of error becomes especially important for the sitting and standing positions, when the arm is likely to be dependent by the individual's side. However, it has been demonstrated that, even with a patient in the supine position, an error of $5 \mathrm{mmHg}$ for diastolic pressure may occur if the arm is not supported at heart level [20-23]. Arm position has become an important issue for self measurement of blood pressure with the manufacture of devices for measuring blood pressure at the wrist, which are proving very popular because of the ease of measurement. Many of these devices are inherently inaccurate, but measurement is even less accurate if the wrist is not held at heart level during measurement.

\section{Which arm?}

The issue as to which arm should be used for blood pressure measurement has been controversial, as some studies using simultaneous measurement have demonstrated significant differences between arms [20-23] The fact that blood pressure differences between arms may be variable makes the issue even more problematic, and differences between sequential arm blood pressure measurements may therefore reflect blood pressure variability and measurement errors. However, a recent study has shown significant differences in inter-arm differences for systolic and diastolic blood pressure [28], leading to the recommendation that bilateral measurement should be made on first consultation and, if reproducible differences greater than $20 \mathrm{mmHg}$ for systolic or $10 \mathrm{mmHg}$ for diastolic pressure are present on consecutive readings, the patient should be referred to a cardiovascular centre for further evaluation with simultaneous bilateral measurement and the exclusion of arterial disease [29].

\section{The cuff and bladder}

The cuff is an inelastic cloth that encircles the arm and encloses the inflatable rubber bladder. It is secured round the arm most commonly by means of Velcro on its adjoining surfaces, occasionally by wrapping a tapering end into the encircling cuff, and rarely by hooks. Tapering cuffs should be long enough to encircle the arm several times: the full length should extend beyond the end of the inflatable bladder for $25 \mathrm{~cm}$ and then should gradually taper for a further $60 \mathrm{~cm}$ [21]. Velcro surfaces must be effective, and when they lose their grip the cuff should be discarded. It should be possible to remove the bladder from the cuff so that the latter can be washed from time to time [21].

'Cuff hypertension'. However sophisticated a blood pressure measuring device may be, if it is dependent on cuff 
occlusion of the arm (as are the majority of devices), it will be prone to the inaccuracy induced by miscuffing, whereby a cuff contains a bladder that is either too long or too short relative to arm circumference. A review of the literature on the century-old controversy relating to the error that may be introduced to blood pressure measurement by using a cuff with a bladder of inappropriate dimensions for the arm has shown that miscuffing is a serious source of error, which inevitably leads to incorrect diagnosis in practice and erroneous conclusions in hypertension research (Table 1). There is unequivocal evidence that either too narrow or too short a bladder (undercuffing) will cause overestimation of blood pressure - so-called 'cuff hypertension' - and there is growing evidence that too wide or too long a bladder (overcuffing) may cause underestimation of blood pressure. Undercuffing has the effect in clinical practice of overdiagnosing hypertension, and overcuffing leads to hypertensive individuals being diagnosed as normotensive. Either eventuality has serious implications for the epidemiology of hypertension and clinical practice [30].

A further problem is that cuff inflation itself may cause a transient but substantial increase (of up to $40 \mathrm{mmHg}$ ) in blood pressure. With SBPM, there may be a transient increase in systolic blood pressure caused by the muscular act of inflating the cuff [31].

Solutions to the problem. A review of the literature shows that a number of approaches have been used over the years to cope with the difficulty of mismatching, and yet none has been ideal [30]. These have included:

- Application of correction factors: Correction formulae to adjust measurement errors derived from the use of an inappropriate bladder have been recommended, but this option has the disadvantage of further complicating the procedure of blood pressure measurement and it has not found acceptance, even when incorporated as a correction band in the standard cuff.

- A range of cuffs: Many national bodies have recommended a range of cuffs to cater for all eventualities. However, this solution presupposes that the user will first measure the arm circumference

Table 1 Mismatching of bladder and arm

\begin{tabular}{|c|c|}
\hline Mismatch & Consequence \\
\hline Bladder too small (undercuffing) & $\begin{array}{l}\text { Overestimation of BP } \\
\text { Range of error } 3.2 / 2.4-12 / 8 \mathrm{mmHg} \\
\text { (as much as } 30 \mathrm{mmHg} \text { in obesity) } \\
\text { More common than overcuffing }\end{array}$ \\
\hline Bladder too large (overcuffing) & $\begin{array}{l}\text { Underestimation of BP } \\
\text { Range of error } 10-30 \mathrm{mmHg}\end{array}$ \\
\hline
\end{tabular}

BP, blood pressure. and that, having done so, will have available an adequate range of cuffs. In practice, neither of these requirements is easily fulfilled.

- Cuffs containing a variety of bladders: A cuff containing three inflatable bladders of varying dimensions has been designed to permit the choice of the most suitable bladder for the arm in which pressure is being measured (Tricuff, Pressure Group AB \& Co KB, AJ Medical HB, Sweden). This cuff, although improving the accuracy of measurement, has a number of disadvantages, which include cost, the difficulty of applying the cuff in routine practice and the stiffness of the cuff.

A cuff for the majority of arms. In an effort to overcome the problem of having a large selection of bladders available, the BHS and the British Standards Institution recommended a cuff containing a bladder measuring $35 \times 12 \mathrm{~cm}$, on the basis that such a bladder would encircle the majority of adult arms. This recommendation presupposed, however, that an error would not be introduced by overcuffing of lean arms. A review of the literature suggests that this presupposition is no longer tenable, although the degree of error remains to be quantified [30]. Because of the problem of overcuffing in thin arms, the BHS now recommends three cuffs [20]:

- a standard cuff with a bladder measuring $12 \times 26 \mathrm{~cm}$ for the majority of adult arms

- a large cuff with a bladder measuring $12 \times 40 \mathrm{~cm}$ for obese arms

- a small cuff with a bladder measuring $12 \times 18 \mathrm{~cm}$ for lean adult arms and children,

whereas the American Heart Association recommends four cuffs [32]:

- a small adult cuff with a bladder measuring $10 \times 24 \mathrm{~cm}$ for arm circumference $22-26 \mathrm{~cm}$

- an adult cuff with a bladder measuring $13 \times 30 \mathrm{~cm}$ for arm circumference $27-34 \mathrm{~cm}$

- a large adult cuff with a bladder measuring $16 \times 38 \mathrm{~cm}$ for arm circumference $35-44 \mathrm{~cm}$

- an adult thigh cuff with a bladder measuring $20 \times$ $42 \mathrm{~cm}$ for arm circumference $45-52 \mathrm{~cm}$.

The 'adjustable cuff'. On the basis of a thorough examination of the literature and in the light of the advances in cuff design, the design features for an 'adult cuff' that would be applicable to all adult arms have been proposed [30]. A.C. Cossor \& Sons Ltd (London) have manufactured the 'Adjustacuff', which, by means of a clamping mechanism on an inflatable bladder, allows the bladder to encircle all arms regardless of arm circumference; to date, experience with this cuff in clinical practice has been limited [9]. 


\section{Factors common to the individual}

Certain groups of people merit special consideration for blood pressure measurement, because of age, body habitus, or disturbances of blood pressure related to haemodynamic alterations in the cardiovascular system. Although there is evidence that many subgroups of the hypertensive population may have peculiarities affecting the accuracy of measurement - such as patients with renal disease, patients with diabetes mellitus, women with pre-eclampsia and youths with 'spurious' hypertension - discussion will be confined to children, the elderly, obese individuals, pregnant women and patients receiving antihypertensive drugs.

\section{Children}

Blood pressure measurement in children presents a number of difficulties, and variability of blood pressure is greater than in adults; thus any one reading is less likely to represent the true blood pressure. In addition, increased variability confers a greater tendency for regression towards the mean. Conventional sphygmomanometry is recommended for general use, but systolic pressure is preferred to diastolic pressure because of greater accuracy and reproducibility. Cuff dimensions are most important, and three cuffs with bladders measuring $4 \times 13 \mathrm{~cm}, 8 \times 18 \mathrm{~cm}$ and the adult dimensions $12 \times 26 \mathrm{~cm}$ are required for the range of arm sizes likely to be encountered in the age range $0-14$ years. The widest cuff practicable should be used. Korotkoff sounds are not reliably audible in all children younger than 1 year, and in many younger than 5 years. In such cases, conventional sphygmomanometry is impossible and more sensitive methods of detection such as Doppler, ultrasound or oscillometry must be used [33]. Ideally, blood pressure should be measured after the child has taken a few minutes of rest. Values obtained during sucking, crying or eating will not be representative. As with adults, a child's blood pressure status should be decided only after it has been measured on a number of separate occasions. Body size is the most important determinant of blood pressure in childhood and adolescence, and the US National High Blood Pressure Education Group on Hypertension Control in Children and Adolescents provides figures for blood pressure ranges related to both age and height [34].

\section{Elderly people}

In epidemiological and interventional studies, blood pressure predicts morbidity and mortality in elderly people as effectively as in the young [35]. The elderly are subject to considerable blood pressure variability; this can lead to a number of diurnal blood pressure patterns, which are best identified using ABPM (see Part III). The practical clinical consequence of these variable patterns in the elderly is that blood pressure measuring techniques can be inaccurate, misleading, or both.
Isolated systolic hypertension. The most common form of hypertension in the elderly is isolated systolic hypertension. The results of the ambulatory study of the Systolic Hypertension in Europe Trial showed that systolic blood pressure measured conventionally in the elderly may average $20 \mathrm{mmHg}$ more than daytime ambulatory blood pressure, thereby leading to inevitable overestimation of isolated systolic hypertension in this group, and probable excessive treatment of the condition [36].

Autonomic failure. The elderly can also show striking variability of blood pressure, with periods of hypotension interspersed with hypertension on ABPM. This pattern, which is often indicative of autonomic failure, is important to identify, so that treatment can be tailored to take account of such fluctuations in blood pressure [36]. As the elderly can be particularly susceptible to the adverse effects of blood pressure lowering drugs, identification of hypotension becomes particularly important.

White-coat hypertension. Even more than the young, the elderly are affected by the white-coat phenomenon (see Part III).

Postural and postprandial hypotension. Postural hypotension is more common in the elderly [37]. It is possible for it to coexist with increased supine and sitting blood pressure, and it is important that blood pressure is assessed in these positions in addition to standing, on initial assessment and from time to time if drugs known to cause postural hypotension are being taken. These include, not only blood pressure decreasing drugs such as diuretics, but also non-cardiovascular drugs, for example neuroleptic agents and tricyclic antidepressants.

Some elderly patients experience quite a marked blood pressure decrease after eating, and this may be symptomatic [38]. Again, this can only be diagnosed definitively by measuring blood pressure with the person standing, after a meal, or using ABPM.

Pseudohypertension. It has been postulated that, as a consequence of the decrease in arterial compliance and arterial stiffening with ageing, indirect sphygmomanometry becomes inaccurate. This has led to the concept of 'pseudohypertension' to describe patients with a large discrepancy between cuff and direct blood pressure measurement [39]. The significance of this phenomenon has been disputed [40], but in elderly patients in whom blood pressure measured with the conventional technique seems to be out of proportion to the clinical findings, referral to a specialist cardiovascular centre for further investigation may be appropriate.

\section{Obese people}

The association between obesity and hypertension has 
been confirmed in many epidemiological studies, and has at least two components [41]. First, there appears to be a pathophysiological connection, and it may well be that in some cases the two conditions are causally linked; secondly, if not taken into account, obesity may result in inaccurate blood pressure values being obtained by indirect measurement techniques. In this regard, it is not yet established whether the accuracy of measurements differs between the oscillometric and the auscultatory methods in this population.

Obesity may affect the accuracy of blood pressure measurement in children, young people, the elderly and pregnant women. The relationship of arm circumference and bladder dimensions has been discussed above. If the bladder is too short, blood pressure will be overestimated ('cuff hypertension'), and if it is too long, blood pressure may be underestimated [30].

\section{Arrhythmias}

The difficulty in measuring blood pressure in patients with arrhythmias is that, when cardiac rhythm is irregular, there is a large variation in blood pressure from beat to beat. Thus, in arrhythmias such as atrial fibrillation, stroke volume - and as a consequence blood pressure - varies depending on the preceding pulse interval. In addition, in such circumstances, there is no generally accepted method of determining auscultatory endpoints. Furthermore, blood pressure measuring devices vary greatly in their ability to record blood pressure accurately in patients with arrhythmias, indicating that devices should be validated independently in patients with arrhythmias [42].

Some physicians may use the first Korotkoff sound as systolic pressure in the presence of arrhythmias, whereas others may equate systolic pressure with the consistent presence of sounds. A similar problem pertains to interpreting diastolic blood pressure. If the first appearance of sound and the final disappearance of all sounds are taken to be systolic and diastolic blood pressure, respectively, it seems likely that the systolic pressure will be overestimated and the diastolic values will be too low. Irrespective of what guidelines are agreed upon, blood pressure measurement in atrial fibrillation, particularly when the ventricular rhythm is highly irregular, will at best constitute a rough estimate, the validity of which can perhaps be improved upon only by using repeated measurements or direct intraarterial measurement. Because the oscillometric method depends on a smooth profile of successive pressure waves to determine blood pressure, this technique is not always able to measure blood pressure in patients with arrhythmias, such as atrial fibrillation. The deflation rate should be no faster than $2 \mathrm{mmHg}$ per heartbeat, and repeated measurements may be required to overcome beat-to-beat variability.
When a bradyarrhythmia is present, there are two potential sources of error: first, if the rhythm is irregular the same problems as with atrial fibrillation will apply; secondly, when the heart rate is extremely slow, for example 40 beats $/ \mathrm{min}$, it is important that the deflation rate used is less than for normal heart rates, as too rapid deflation will lead to underestimation of systolic and overestimation of diastolic pressure.

\section{Pregnancy}

Clinically relevant hypertension occurs in more than $10 \%$ of pregnant women in most populations, and in a significant number of these increased blood pressure is a key factor in medical decision-making in the pregnancy. Particular attention must be paid to blood pressure measurement in pregnancy, because of the important implications for patient management, in addition to the fact that it presents some special problems [43] (see also Part III). There has been much controversy as to whether the muffling or disappearance of sounds should be taken for diastolic blood pressure. The general consensus from obstetricians, based on careful analysis of the evidence, is that disappearance of sounds (fifth phase) is the most accurate measurement of diastolic pressure except when sounds persist to zero, when the fourth phase of muffling of sounds should be used $[44,45]$.

\section{Patients receiving antihypertensive medication}

In patients receiving treatment with antihypertensive drugs, the timing of measurement may have a substantial influence on the blood pressure however it is measured, and it is recommended that the time of drug ingestion is noted, so as to indicate the expected trough or peak levels of drug effect.

\section{Exercise blood pressure}

Systolic blood pressure increases with increasing dynamic work as a result of increasing cardiac output, whereas diastolic pressure usually remains about the same or moderately lower. Normal values of maximum systolic blood pressure for men have been defined and are directly related to age [46]. However, these normal values do not take account of the fact that resting blood pressure increases with increasing age. If normal values for exercise systolic blood pressure are determined on the basis of the corresponding percentiles of a resting systolic blood pressure of $140 \mathrm{mmHg}$, age-independent upper limits of normality can be derived. An ageindependent upper limit of $180 \mathrm{mmHg}$ systolic for exercise blood pressure at $100 \mathrm{~W}$ standardized bicycle test has been proposed [47].

There is evidence that an exaggerated blood pressure response with exercise testing may be predictive of future hypertension [48-50] and future mortality from myocardial infarction [51], and may be associated with 
angiographic coronary artery disease [52]. Furthermore, normotensive individuals with an exaggerated blood pressure response during exercise (e.g. systolic blood pressure during peak exercise of $210 \mathrm{mmHg}$ or greater) may be at high risk of developing left ventricular hypertrophy [53]. Moreover, it has been shown that, in patients with hypertension, left ventricular mass index is poorly related to blood pressure at rest, but is related to exercise systolic blood pressure [54]. Several studies based on non-invasive methods have concluded that exercise-induced increases in blood pressure during an ergometer bicycle test predict future mortality and cardiovascular complications $[55,56]$.

The evidence from these studies has led inevitably to the question as to whether exercise blood pressure should be used in routine clinical practice. Several issues need to be addressed, however, before such a conclusion can be accepted. Although systolic blood pressure may be recorded reasonably well during bicycle exercise by conventional sphygmomanometry, diastolic values may be grossly overestimated or underestimated. This is also true for the automatic blood pressure devices using either the auscultatory or oscillometric techniques. With correct use of conventional sphygmomanometry, systolic values are reasonably accurate when the arm used for measurement is kept steady. Furthermore, prognostic data are mainly available for systolic blood pressure during exercise and it would be reasonable, therefore, to use only systolic values in clinical practice. Most of the prognostic studies were based on bicycle exercise tests. The use of the ergometer bicycle allows standardization of the procedure, such as exercise time and an exact determination of the exercise load. In some studies, the blood pressure at fixed workload was used, but with fixed workloads patients may be performing at different percentages of their maximal exercise capacity.

At present, the additional information provided by exercise blood pressure does not justify its use in routine clinical practice. However, exercise testing is performed for many indications other than hypertension. When an exaggerated blood pressure response to exercise is seen in normotensive and hypertensive individuals, it should not be ignored, but seen rather as providing important prognostic information. In this context, exercise systolic blood pressure values greater than $200 \mathrm{mmHg}$ at a moderate workload (600 k.p.m./min; $100 \mathrm{~W}$ ) during standardized bicycle test should be regarded as abnormal.

\section{Part II. Conventional sphygmomanometry Basic requirements for auscultatory blood pressure measurement}

The auscultatory technique is being replaced by automated techniques, and its place in medicine may soon be of historical rather than practical interest. The technique has been the subject of recent reviews and only a summary of the procedure will be presented here $[20,57]$.

The measurement of blood pressure in clinical practice by the century-old technique of Riva-Rocci/Korotkoff is dependent on the accurate transmission and interpretation of a signal (Korotkoff sound or pulse wave) from an individual via a device (the sphygmomanometer) to an observer. Errors in measurement can occur at each of these points of interaction, but by far the most fallible component is the observer.

\section{Observer error}

In 1964, Geoffrey Rose and his colleagues classified observer error into three categories [58]:

(i) systematic error that leads to both intraobserver and interobserver error

(ii) terminal digit preference, which results in the observer rounding off the pressure reading to a digit of his or her choosing, most often to zero

(iii) observer prejudice or bias, whereby the observer simply adjusts the pressure to meet his or her preconceived notion of what the pressure should be.

Training observers in the technique of auscultatory blood pressure measurement is often taken for granted $[59,60]$. Instruction to medical students and nurses has not always been as comprehensive as it might be, and assessment for competence in measuring blood pressure has been a relatively recent development. Training methods have included: direct instruction using a binaural or multiaural stethoscope, the use of manuals, booklets and published recommendations, audio-tape training methods, video-films and, recently, CD-ROMs that incorporate instruction with examples of blood pressure measurement using a falling mercury column with Korotkoff sounds and a means for the student to assess competence in the technique using a series of examples [20-23]. To eliminate observer error completely requires a period of intensive training, repeated at regular intervals if necessary, and it would be unrealistic to expect that such standards would be implemented in clinical practice. The criteria for observer accuracy are necessarily more stringent for research work than for clinical practice, and recommendations for observer training have been drawn up for both [60].

The London School of Hygiene sphygmomanometer and the Hawksley 'zero-muddler' sphygmomanometer were designed to minimize observer error in research and, although popular for many years, neither is much used now because the design modifications introduced inaccuracies [61-63]. 


\section{Mercury and aneroid sphygmomanometers}

The mercury sphygmomanometer is a reliable device, but all too often its continuing efficiency has been taken for granted, whereas the aneroid manometer, which is not generally as accurate, is often assumed to be as reliable. These devices have certain features in common: each has an inflation/deflation system and an occluding bladder encased in a cuff, and both devices measure blood pressure by auscultation with a stethoscope [57].

Inflation/deflation system. The inflation/deflation system consists of an inflating and deflating mechanism connected by rubber tubing to an occluding bladder. The standard mercury and aneroid sphygmomanometers used in clinical practice are operated manually, with inflation being effected by means of a bulb compressed by hand and deflation by means of a release valve, which is also controlled by hand. The pump and control valve are connected to the inflatable bladder and thence to the sphygmomanometer by rubber tubing. Leaks from cracked or perished rubber make accurate measurement of blood pressure difficult because the fall of the mercury cannot be controlled; the rubber should be in a good condition and free from leaks. The length of tubing between the cuff and the manometer should be at least $70 \mathrm{~cm}$ and that between the inflation source and the cuff should be at least $30 \mathrm{~cm}$. Connections should be airtight and easily disconnected [57].

Controlvalve. One of the most common sources of error in sphygmomanometers is the control valve. Defective valves cause leakage, making control of pressure release difficult; this leads to underestimation of systolic and overestimation of diastolic pressures (Table 2).

Hasards of mercury. The mercury sphygmomanometer is a simple and accurate device that can be easily serviced, but there are rightly concerns about the toxicity of mercury for individuals using mercury sphygmomanometers, and for those who have to service them. Users should therefore be alert to the hazards associated with handling mercury. However, the greatest concern about mercury is its toxic effects on the environment.
The mercury thermometer has been replaced in many countries, and in Sweden and the Netherlands the use of mercury is no longer permitted in hospitals. However, in other European countries, including the UK and Ireland, the move to ban mercury from hospital use has not been received with enthusiasm, on the grounds that we do not have an accurate alternative to the mercury sphygmomanometer. None the less, the fear of mercury toxicity is making it difficult to have mercury sphygmomanometers serviced, and the precautions recommended for dealing with a mercury spill are influencing purchasing decisions. Indeed, this is what central governmental policy in many countries would favour - the gradual disappearance of mercury from clinical use, rather than imposition of a ban [64-67].

Banning mercury from the wards raises another issue, which may be of even greater importance for clinical medicine. If the millimetre of mercury is no longer the unit of measurement for blood pressure, there can be no scientific argument against its replacement with the Système International (SI) unit, the kilopascal, which is the accepted unit of pressure measurement elsewhere in science [68].

Maintenance. Mercury sphygmomanometers are easily checked and maintained, but great care should be taken when handling mercury. The revised European Standard recommends that mercury sphygmomanometers display a warning to this effect [12-14]. Mercury sphygmomanometers need cleaning and checking at least every 6 months in hospital use, and every 12 months in general use [57]. As many as 50\% of sphygmomanometers in hospital use are defective, and a maintenance policy should be mandatory in all hospitals [69]; few have any such policy. Doctors in practice also often neglect to have their sphygmomanometers checked and serviced. The responsibility for reporting faulty equipment or lack of appropriate cuffs lies with the observer, who should always refuse to use defective or inappropriate equipment.

\section{Aneroid manometers}

Aneroid sphygmomanometers register pressure through

Table 2 Features affecting accuracy of the mercury sphygmomanometer

- The top of the mercury meniscus should rest at exactly zero without pressure applied; if it is below, add mercury.

- The scale should be clearly calibrated in $2 \mathrm{~mm}$ divisions from 0 to $300 \mathrm{mmHg}$ and should indicate accurately the differences between the levels of mercury in the tube and in the reservoir.

- The diameter of the reservoir must be at least 10 times that of the vertical tube, or the vertical scale must correct for the drop in the mercury level in the reservoir as the column rises.

- Substantial errors may occur if the manometer is not kept vertical during measurement. Calibrations on floor models are especially adjusted to compensate for the tilt in the face of the gauge. Stand-mounted manometers are recommended for hospital use. This allows the observer to adjust the level of the sphygmomanometer and to perform measurement without having to balance the sphygmomanometer precariously on the side of the bed.

- The air vent at the top of the manometer must be kept patent, as clogging will cause the mercury column to respond sluggishly to pressure changes and to overestimate pressure.

- The control valve is one of the most common sources of error in sphygmomanometers and when it becomes defective it should be replaced. Spare control valves should be available in hospitals and a spare control valve should be supplied with sphygmomanometers. 
a bellows and lever system, which is mechanically more intricate than the mercury reservoir and column [57]. The jolts and bumps of everyday use affect accuracy over time, usually leading to false-low readings, with the consequent underestimation of blood pressure. They are therefore less accurate in use than mercury sphygmomanometers. When aneroid instruments are calibrated against a mercury sphygmomanometer, a mean difference of $3 \mathrm{mmHg}$ is considered to be acceptable; however, $58 \%$ of aneroid sphygmomanometers have been shown to have errors greater than $4 \mathrm{mmHg}$, with about $33 \%$ of these having errors greater than $7 \mathrm{mmHg}$ [70]. Moreover, aneroid sphygmomanometry is prone to all the problems of the auscultatory technique, namely observer bias and terminal digit preference.

Aneroid sphygmomanometers should be checked every 6 months against an accurate mercury sphygmomanometer over the entire pressure range. This can be achieved by connecting the aneroid sphygmomanometer, via a Y-piece, to the tubing of the mercury sphygmomanometer and inflating the cuff around a bottle or cylinder. If inaccuracies or other faults are found, the instrument must be returned to the manufacturer or supplier for repair [57].

As mercury sphygmomanometers are removed from clinical practice, there is a tendency to replace them with aneroid devices on the false assumption that, because each can be used to measure blood pressure using the auscultatory technique, one can be interchanged for the other. There is remarkably little literature on the accuracy of aneroid devices and what does exist is generally negative. Perhaps the broader view might be that, because the auscultatory technique itself is inherently inaccurate whether the mercury or aneroid sphygmomanometer is used, the sooner accurate automated techniques of measurement are introduced, the better.

\section{Stethoscope}

A stethoscope should be of a high quality, with clean, well-fitting earpieces. The American Heart Association recommends using the bell of the stethoscope over the brachial artery, rather than placing the diaphragm over the antecubital fossa, on the basis that the bell is most suited to the auscultation of low-pitched sounds, such as the Korotkoff sounds. However, it probably does not matter much if the bell or diaphragm is used in routine blood pressure measurement, provided the stethoscope is placed over the palpated brachial artery in the antecubital fossa. As the diaphragm covers a greater area and is easier to hold than a bell endpiece, it is reasonable to recommend it for routine clinical measurement of blood pressure [57].

\section{Non-automated alternatives to the mercury sphygmomanometer}

The oscillometric method of blood pressure measurement has a number of inherent limitations, which make it unlikely that it will ever be acceptable as the gold standard for CBPM. The need for accurate clinic measurement will always be present, and the fact that important clinical decisions will continue to be made on very small numbers of readings (often one, and rarely more than three) emphasizes the need for maximal accuracy.

An alternative to the mercury sphygmomanometer is the so-called 'hybrid' sphygmomanometer, which combines some of the features of both the electronic and mercury devices. It uses an electronic pressure gauge as a substitute for the mercury column. Blood pressure is taken in the same way as with a mercury device, using a stethoscope and listening for the Korotkoff sounds. The cuff pressure is displayed as a simulated mercury column using an array of LCDs, and also as a digital LCD readout. The cuff is deflated in the normal way and, when systolic and diastolic pressure are heard, a button next to the deflation knob is pressed, which freezes the digital display to show systolic and diastolic pressures, thus offering the potential of eliminating terminal digit preference, which is a major problem with the clinical use of any auscultatory monitor. Working prototypes have been developed, but the device is not yet commercially available [71]. A similar concept using an electronic transducer instead of the mercury column has been developed by A.C. Cossar and Son (London, UK). Known as the 'Greenlight 300', this device is available commercially, but has not yet been validated. With such devices, the physician is still able to measure blood pressure using the traditional auscultatory technique, without having necessarily to rely on automated readings, and this is achieved without the problems associated with mercury columns or aneroid devices.

\section{Automated alternatives to the mercury sphygmomanometer} An accurate automated sphygmomanometer capable of providing printouts of systolic, diastolic and mean blood pressure, together with heart rate and the time and date of measurement, should eliminate errors of interpretation and abolish observer bias and terminal digit preference. Moreover, the need for elaborate training as described above would no longer be necessary, although a period of instruction and assessment of proficiency in using the automated device will always be necessary. Another advantage of automated measurement is the ability of such devices to store data for later analysis. This development is in fact taking place, and a number of large research studies are using automated technology to measure blood pressure instead of the traditional mercury 'gold standard'. For 
example, in the large Anglo-Scandinavian Cardiac Outcome Trial [72], the validated Omron HEM-705CP automated monitor was used [73].

Although the mercury sphygmomanometer is disappearing from use, unfortunately there are not many alternative devices available to replace it. In addition, the advent of accurate automated devices, however welcome, is not without problems [64]. First, automated devices have been notorious for their inaccuracy, although accurate devices are now appearing on the market. Secondly, most of the available automated devices were designed for self-measurement of blood pressure, and it should not be assumed that they will be suitable for clinical use, or that they will remain accurate with use, although some are being used successfully in hospital practice. Thirdly, oscillometric techniques cannot measure blood pressure in all situations, particularly in patients with arrhythmias such as atrial fibrillation with a rapid ventricular response, and there are also individuals in whom these devices cannot measure blood pressure, for reasons that are not always apparent. Fourthly, doctors are uneasy about trusting algorithmic methods, which are guarded zealously by manufacturers. To ensure that new devices conform with recommended validation protocols, the mercury sphygmomanometer will have to be retained as a gold standard in designated laboratories.

\section{Performing auscultatory measurement Position of manometer}

The observer should take care about positioning the manometer:

- The manometer should be no further than $1 \mathrm{~m}$ away, so that the scale can be read easily.

- The mercury column should be vertical (some models are designed with a tilt) and at eye level. This is achieved most effectively with standmounted models, which can be easily adjusted to suit the height of the observer.

- The mercury manometer has a vertical scale and errors will occur unless the eye is kept close to the level of the meniscus. The aneroid scale is a composite of vertical and horizontal divisions and numbers, and must be viewed straight-on, with the eye on a line perpendicular to the centre of the face of the gauge.

\section{Placing the cuff}

The cuff should be wrapped round the arm, ensuring that the bladder dimensions are accurate. If the bladder does not completely encircle the arm, its centre must be over the brachial artery. The rubber tubes from the bladder are usually placed inferiorly, often at the site of the brachial artery, but it is now recommended that they should be placed superiorly or, with completely encircling bladders, posteriorly, so that the antecubital fossa is easily accessible for auscultation. The lower edge of the cuff should be $2-3 \mathrm{~cm}$ above the point of brachial artery pulsation [20,57].

\section{Palpatory estimation of blood pressure}

The brachial artery should be palpated while the cuff is rapidly inflated to about $30 \mathrm{mmHg}$ above the point at which the pulse disappears; the cuff is then slowly deflated, and the observer notes the pressure at which the pulse reappears. This is the approximate level of the systolic pressure. Palpatory estimation is important, because phase I sounds sometimes disappear as pressure is reduced and reappear at a lower level (the auscultatory gap), resulting in systolic pressure being underestimated unless already determined by palpation. The palpatory technique is useful in patients in whom auscultatory endpoints may be difficult to judge accurately: for example, pregnant women, patients in shock or those taking exercise. (The radial artery is often used for palpatory estimation of the systolic pressure, but by using the brachial artery the observer also establishes its location before auscultation.)

\section{Auscultatory measurement of systolic and diastolic pressures}

- Place the stethoscope gently over the brachial artery at the point of maximal pulsation; a bell endpiece gives better sound reproduction, but in clinical practice a diaphragm is easier to secure with the fingers of one hand, and covers a larger area.

- The stethoscope should be held firmly and evenly but without excessive pressure, as too much pressure may distort the artery, producing sounds below diastolic pressure. The stethoscope endpiece should not touch the clothing, cuff or rubber tubes, to avoid friction sounds.

- The cuff should then be inflated rapidly to about $30 \mathrm{mmHg}$ above the palpated systolic pressure and deflated at a rate of $2-3 \mathrm{mmHg}$ per pulse beat (or per second), during which the auscultatory phenomena described below will be heard.

- The phases shown in Table 3, which were first described by Nicolai Korotkoff and later elaborated by Witold Ettinger, may now be heard [74].

- When all sounds have disappeared, the cuff should be deflated rapidly and completely to prevent venous congestion of the arm before the measurements is repeated.

\section{Diastolic dilemma}

For many years, recommendations on blood pressure measurement have been equivocal as to the diastolic endpoint - the so-called 'diastolic dilemma'. Phase IV (muffling) may coincide with, or be as much as, $10 \mathrm{mmHg}$ greater than phase $\mathrm{V}$ (disappearance), but usually the difference is less than $5 \mathrm{mmHg}$. There has 
Table 3 Auscultatory sounds

\begin{tabular}{ll}
\hline Phase I & $\begin{array}{l}\text { The first appearance of faint, repetitive, clear tapping sounds that gradually increase in } \\
\text { intensity for at least two consecutive beats is the systolic blood pressure. }\end{array}$ \\
$\begin{array}{l}\text { Phase II } \\
\text { Auscultatory gap brief period may follow during which the sounds soften and acquire a swishing quality. } \\
\text { In some patients, sounds may disappear altogether for a short time. } \\
\text { Phase III }\end{array}$ & $\begin{array}{l}\text { The return of sharper sounds, which become crisper, to regain or even exceed the } \\
\text { intensity of phase I sounds. }\end{array}$ \\
Phase IV & $\begin{array}{l}\text { The distinct, abrupt muffling of sounds, which become soft and blowing in quality. } \\
\text { Phase V }\end{array}$
\end{tabular}

The clinical significance, if any, of phases II and III has not been established.

been resistance to general acceptance of the silent endpoint until recently, because the silent endpoint can be considerably below the muffling of sounds in some groups of patients, such as children, pregnant women, or anaemic or elderly patients. In some patients, sounds may even be audible when cuff pressure is deflated to zero. There is now a general consensus that disappearance of sounds (phase V) should be taken as diastolic pressure (as originally recommended by Korotkoff in 1910) [74]. When the Korotkoff sounds persist down to zero, muffling of sounds (phase IV) should be recorded for diastolic pressure, and a note made to this effect.

\section{Recording blood pressure}

The following points should be recorded with the blood pressure measurement [made to the nearest $2 \mathrm{mmHg}$ without rounding-off to the nearest 5 or $10 \mathrm{mmHg}$ (digit preference)]:

(i) position of the individual - lying, sitting or standing

(ii) the arm in which the measurement will be made - right or left

(iii) blood pressure in both arms on first attendance

(iv) arm circumference and inflatable bladder size

(v) phases IV and V for diastolic blood pressure

(vi) an auscultatory gap if present

(vii) state of the individual - e.g. anxious, relaxed

(viii) time of drug ingestion.

Number of measurements. Because of the variability of measurements of casual blood pressure, decisions based on single measurements will result in erroneous diagnosis and inappropriate management. Reliability of measurement is improved if repeated measurements are made. At least two measurements at $1 \mathrm{~min}$ intervals should be taken carefully at each visit, with a repeat measurement if there is uncertainty or distraction; it is best to make a few carefully taken measurements rather than taking a number of hurried measurements.

The alarm reaction to blood pressure measurement may persist after several visits, so for patients in whom sustained increases of blood pressures are being assessed, a number of measurements should be made on different occasions over a number of weeks or months before diagnostic or management decisions are made. Alternatively, ABPM or SBPM away from the medical environment should be performed.

\section{Part III. Ambulatory blood pressure measurement}

Ambulatory blood pressure measurement is being used increasingly in clinical practice [75-79]. In recognition of this, a number of national societies have published recommendations for the use and interpretation of ABPM in clinical practice [80-83], and the ESH has published recommendations on blood pressure measuring devices, including devices for ABPM [2]. Importantly, the technique has finally been approved for reimbursement in the USA [84]. It is important to emphasise that the diagnosis of hypertension by whatever measurement technique is only one factor in a patient's risk profile, which must be assessed in its totality, in relation to concomitant disease such as diabetes mellitus, and in relation to the degree of target-organ involvement as recommended in national and international guidelines [85-87]. This section is concerned only with ABPM in adults.

\section{General considerations \\ Why is ABPM superior to conventional blood pressure measurement?}

The evidence that ABPM gives information over and above CBPM has been growing steadily over the past 25 years [75]. There are a number of obvious advantages: the technique gives more measurements than CBPM, and the real blood pressure is reflected more accurately by repeated measurements [78]. ABPM provides a profile of blood pressure away from the medical environment, thereby allowing identification of individuals with a white-coat response [88]. ABPM shows blood pressure behaviour over a 24-h period during the patient's usual daily activities, rather then when they are sitting in the artificial circumstances of a clinic or office; ABPM can demonstrate the efficacy of antihypertensive medication over a $24-\mathrm{h}$ period, which facilitates more rational prescribing than having to base a decision on one or a few CBPMs confined to a short period of the diurnal cycle [78]. ABPM can identify patients whose blood pressure does not reduce at night- 
time - the non-dippers - who are at greater risk for cardiovascular morbidity [89]. Finally, the technique can demonstrate a number of patterns of blood pressure behaviour that may be relevant to clinical management - white-coat hypertension, hypotension, dipping and non-dipping, etc. [90]. These advantages should have brought ABPM into much wider clinical use many years ago. However, this eventuality was delayed because, despite a large body of work showing that ABPM predicted outcome on the basis of surrogate markers such as left ventricular hypertrophy, microalbuminuria and arterial stiffness [78,91-93], there was insufficient prospective evidence to show that ABPM was superior to CBPM in predicting cardiovascular mortality. Another relevant factor was concern, particularly in the USA, that the technique, once approved for reimbursement, would be over-used in practice.

Evidence is now available from longitudinal studies that ABPM is a stronger predictor of cardiovascular morbidity and mortality than CBPM [78,94-99] and, in the USA, the Centers for Medicare and Medicaid Services (CMS) have recently approved ABPM for reimbursement for the identification of individuals with white-coat hypertension [84]. It would seem, therefore, that there is now international acceptance that ABPM is an indispensable investigation in patients with established and suspected hypertension. This recommendation has important implications for clinical practice. Among the questions that need to be answered are:

- Which ABPM devices should be used?

- How should the data be presented?

- How best can doctors and nurses unfamiliar with the technique be educated in its use and interpretation of the data?

- How can the technique be best used to yield the maximum of information without subjecting patients to unnecessary investigation?

- What are the financial implications?

\section{Choosing an ABPM device}

The first step in adopting the technique of ABPM is selecting a device, and the first consideration is to ensure that the device selected is accurate [2]. As has already been stated in Part I, it is acknowledged that the accuracy of blood pressure measuring devices should not be based on claims from manufacturers, which can at times be somewhat extravagant, and independent validation according to the criteria of the BHS, AAMI or ESH protocols, with the results published in peer-reviewed journals, should be demanded [2].

\section{Choosing ABPM software}

Although the BHS protocol does make certain recom- mendations in relation to the software programs used in ABPM devices, too little consideration has been given to this important aspect of the technique. All ABPM devices are sold with individual software packages, which present data in a variety of ways, and in some instances the software programs are neither 'userfriendly' nor inexpensive. It is important, therefore, to be sure that ABPM software programs are suitable for the use for which the device has been chosen. For example, in a busy general practice, perhaps only basic data giving average day- and night-time values and a visual plot are all that will be required, whereas for research purposes statistical detail on the windows of the 24-h profile and indices such as pulse pressure, blood pressure load, coefficient of variation, cusum values, etc., may be required.

The use of ABPM in clinical practice could be facilitated by two developments. First, if the graphic presentation of ABPM data was standardized, much as is the case for electrocardiographic (ECG) recordings, the presentation of data would be independent of the type of ABPM monitor used and the user would not have to become familiar with a variety of programs. Moreover, standardization would facilitate the interchange of ABPM recordings between databases, such as hospital and primary care practice. Secondly, if ABPM software programs could provide a printed report of the ABPM data, as is possible with ECG recordings, doctors and nurses unfamiliar with the technique would be assisted in learning the variety of patterns generated by ABPM and, importantly, the time needed for a physician to report on each ABPM would be greatly reduced, thereby reducing the cost of the technique $[79,83,100]$ (Fig. 1 and Table 4).

\section{Financial considerations}

Analysis of the cost/benefit relationships of ABPM is complex and awaits full study [101]. ABPM is more expensive than CBPM, but the benefits to patients would seem to justify the additional expense. Individuals with white-coat hypertension, which may be present in as many as $25 \%$ of people who appear to have hypertension with conventional measurement, may be spared years of unnecessary and expensive drug treatment [25]. Likewise, ABPM may spare patients with white-coat hypertension being penalized for insurance or employment by having the diagnosis of 'hypertension' misapplied [102]. It has been shown that, when ABPM, rather than clinic blood pressure, is used as the basis for prescribing, significantly less antihypertensive medication is prescribed [103]. The financial saving from less drug prescribing has been analysed in a cost/benefit comparison of ABPM with CBPM in Switzerland. It was estimated that, over a 10-year period, 2 million Swiss francs could be saved if therapeutic decisions were based on ABPM rather than CBPM [104]. 
Fig. 1

(a)

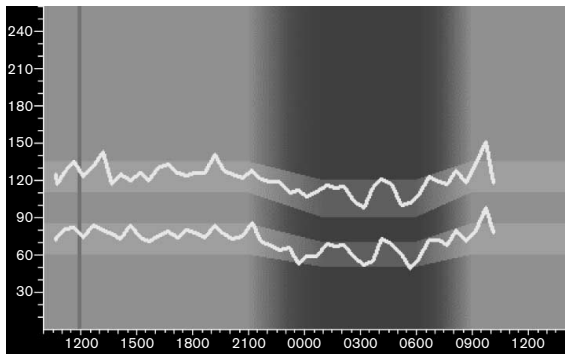

(b)

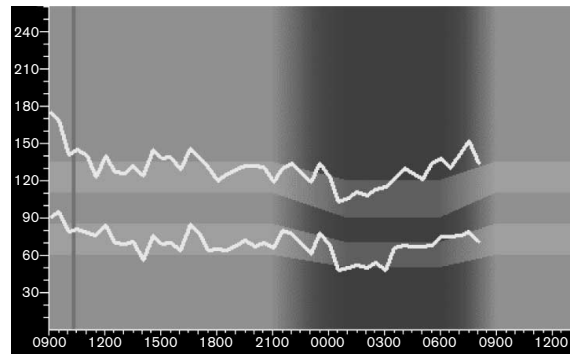

(d)

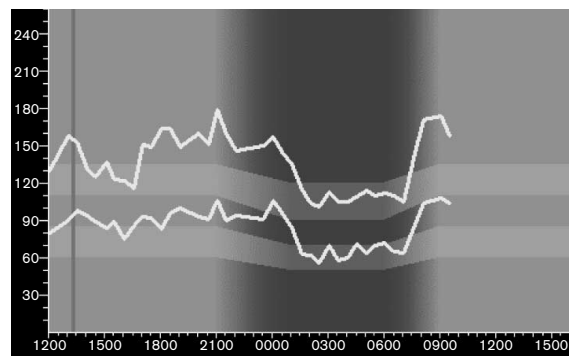

(f)

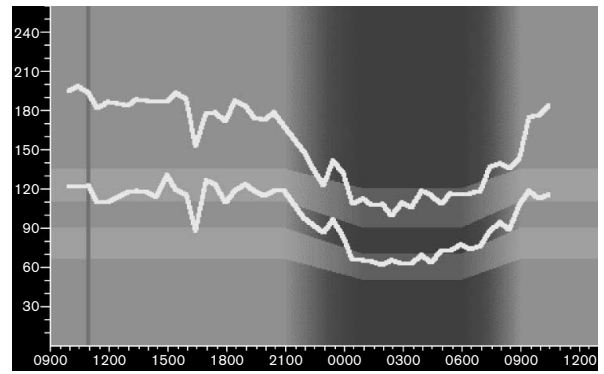

(c)

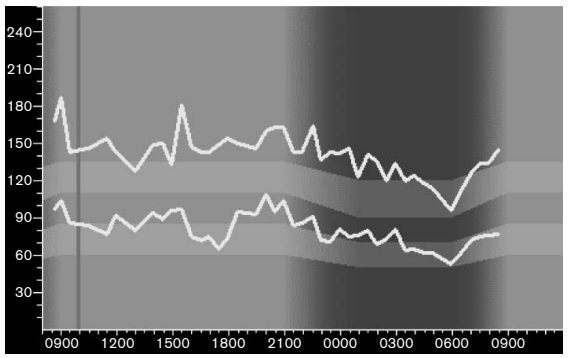

(e)

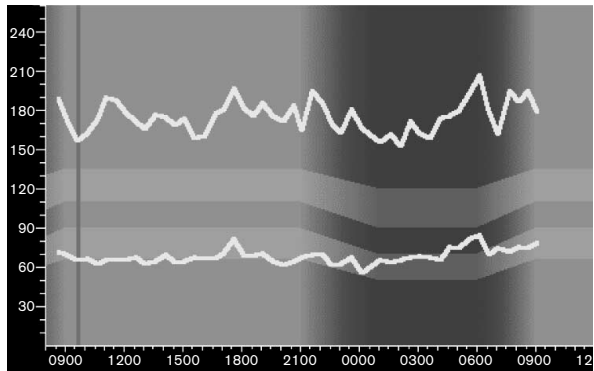

(g)

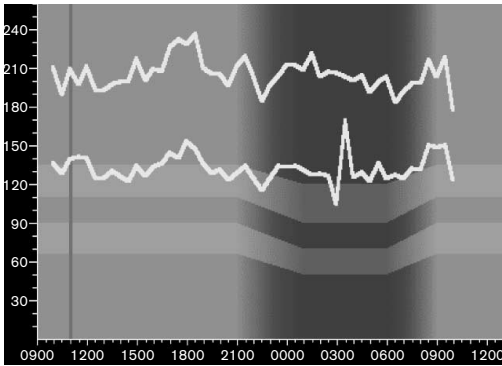

Standardised common patterns of ABPM and reports generated by the dabl ${ }^{\circledR}$ ABPM Program (ECF Medical Ltd, Blackrock, Co. Dublin, Ireland, www.ecfmedical.com). Common to all reports: 'On the basis of the data recorded and the available literature, the ABPM suggests...'. Common to all plots: vertical axes show blood pressures; horizontal axes show 24-h clock times; horizontal bands indicate normal values for 24-h systolic and diastolic blood pressures; shaded vertical areas indicate night-time. (a) Normal ABPM pattern. This ABPM suggests normal 24-h systolic and diastolic blood pressures (128/78 $\mathrm{mmHg}$ daytime, $110 / 62 \mathrm{mmHg}$ night-time). (b) White-coat hypertension. This ABPM suggests white-coat hypertension $(175 / 95 \mathrm{mmHg})$ with otherwise normal $24-\mathrm{h}$ systolic and diastolic blood pressures $(133 / 71 \mathrm{mmHg}$ daytime, $119 / 59 \mathrm{mmHg}$ nighttime). (c) White-coat effect. This ABPM suggests mild daytime systolic hypertension (149 $\mathrm{mmHg})$, borderline daytime diastolic hypertension $(87 \mathrm{mmHg})$, borderline night-time systolic hypertension $(121 \mathrm{mmHg})$ and normal night-time diastolic blood pressures $(67 \mathrm{mmHg})$ with white-coat effect (187/104 mmHg). (d) Systolic and diastolic hypertension. This ABPM suggests mild daytime systolic and diastolic hypertension (147/ $93 \mathrm{mmHg}$ ), but normal night-time systolic and diastolic blood pressures $(111 / 66 \mathrm{mmHg})$. (e) /solated systolic hypertension. This ABPM suggests severe 24 -h isolated systolic hypertension (176/68 mmHg daytime, $169 / 70 \mathrm{mmHg}$ night-time). (f) Hypertensive dipper. This ABPM suggests severe daytime systolic hypertension $(181 \mathrm{mmHg})$, moderate daytime diastolic hypertension $(117 \mathrm{mmHg})$ and normal night-time systolic and diastolic blood pressures $(111 / 68 \mathrm{mmHg})$. (g) Hypertensive non-dipper. This ABPM suggests severe 24-h systolic and diastolic hypertension (210/ $134 \mathrm{mmHg}$ daytime, 205/130 $\mathrm{mmHg}$ night-time). 
Table 4 Ranges for definitions used in the $d a b /{ }^{\circledR}$ ambulatory blood pressure monitoring reports

\begin{tabular}{lcccccc}
\hline & \multicolumn{5}{c}{ Hypertension $(\mathrm{mmHg})$} \\
\cline { 2 - 7 } & Low & Normal & Borderline & Mild & Moderate & Severe \\
\hline Daytime & & & & & & \\
SBP & $<100$ & $100-135$ & $136-140$ & $141-155$ & $156-170$ & $>170$ \\
DBP & $<65$ & $65-85$ & $86-90$ & $91-100$ & $101-110$ & $>110$ \\
Night-time & & & & & & \\
SBP & $<90$ & $91-120$ & $121-125$ & $126-135$ & $136-150$ & $>150$ \\
DBP & $<50$ & $51-70$ & $71-75$ & $76-85$ & $86-100$ & $>100$ \\
\hline
\end{tabular}

SBP, DBP, systolic and diastolic blood pressures.

\section{Training requirements}

The technique of ABPM is a specialized one, and should be approached with the care reserved for any such procedure. An understanding of the principles of traditional blood pressure measurement, cuff fitting, monitor function and analysis, and interpretation of ABPM data is required. In practice, a nurse with an interest and experience in hypertension can master the use of ABPM devices after relatively brief training. However, the analysis and interpretation of ABPM profiles requires experience in the technique, which is best acquired by the doctor in charge of an ABPM service.

\section{Using an ABPM monitor}

Time needs to be given to fitting the monitor and preparing the patient for the monitoring period if good results are to be obtained [75-83]. The key to successful ABPM is educating the patient on the process of monitoring and the instructions should be explained and printed on a diary card (Table 5). In clinical practice, measurements are usually made at 20-30 min intervals in order not to interfere with activity during the day and with sleep at night, but measurements can be made more frequently if indicated. The frequency of measurement during the 24-h period is generally not greater than every $15 \mathrm{~min}$ (which could interfere with normal activity), or less frequent than every $30 \mathrm{~min}$ (which could give an inadequate number of measure-

Table 5 Using an ambulatory blood pressure monitor

- 15-30 min needed

- Relax patient in a quiet room

- Enter patient's details into monitor

- Measure BP in both arms

If SBP difference $<10 \mathrm{mmHg}$, use non-dominant arm

If SBP difference $\geqslant 10 \mathrm{mmHg}$, use arm with greater pressure

- Select appropriate cuff

- Select frequency of measurement - usually every 30 min day and night

- Inactivate LCD display

- Give patient written instructions and a diary card

- Instruct patient how to remove and inactivate monitor after $24 \mathrm{~h}$

BP, blood pressure; SBP, systolic blood pressure. ments). Some workers prefer to select 15-20 min intervals during the day and 30 -min intervals at night, with others preferring to take 30 -min intervals throughout the $24-\mathrm{h}$ period. There should be at least 14 measurements of systolic and diastolic blood pressure during the day, and at least seven measurements at night; if this minimum requirement is not met, the ABPM should be repeated.

There are a number of ways of analysing blood pressures recorded during the 24-h cycle [105,106]. One simple and popular method is to assess the time of awakening and sleeping from diary card entries. Another method is to use a fixed time method in which the retiring (2101-0059h) and rising (0601-0859h) periods (during which blood pressures are subject to considerable variation) are eliminated, with the daytime period being from 0900 to $2100 \mathrm{~h}$ and night-time from 0100 to $0600 \mathrm{~h}$; in this way the variations that may exist between the young and the old and in different cultures are to some extent eliminated from the analysis [105]. This method has the disadvantage of eliminating information on the white-coat window and early morning surge of blood pressure, which may be associated with cardiovascular events [106]. However, these windows can be analysed separately for research or if considered important in practice. In countries where daytime siestas are popular, such as the Mediterranean regions, a record of sleeping times is important and ABPM software should be able to adjust for this [107]. To improve reproducibility, ABPM measurements should be performed on like days, for example on working or recreational days. A diary card may be used to record symptoms and events that may influence ABPM measurements.

\section{Editing ABPM data}

Many statistical techniques exist for describing different aspects of ambulatory records, and no one method is ideal [108]. The detection of artefactual readings and the handling of outlying values (which may or may not be erroneous) have been the subject of debate [106]. Several automatic procedures have been proposed to 
reject outlying measurements, based on a univariate, multivariate or temporal approach. More complex procedures requiring the intervention of the observer in the editing process have been devised, but they are time-consuming and thus not suitable in clinical practice [109]. It has been shown that rejection of outliers may alter the results of ABPM substantially, mainly affecting the average values of systolic ABPM and blood pressure variability [109,110]. Editing procedures can reduce mean systolic ABPM by $4 \mathrm{mmHg}$ in comparison with unedited recordings, thereby decreasing the likelihood of diagnosing sustained hypertension. In one study, the prevalence of sustained hypertension was decreased by $6-10 \%$ when several editing methods were applied to raw 24-h data [110]. Rejection of outliers affects blood pressure variability to a larger extent, with reductions in the standard deviation of daytime systolic blood pressure ranging from 30 to $42 \%$ with the various procedures [110].

To make the results from different laboratories comparable, common editing criteria for ABPM should be used throughout the world. The problem is to establish which of the methods proposed in the literature provides the most reliable data. Whereas some methods seem to provide the best results in terms of ABPM reproducibility [111], others require the intervention of the observer or allow fully automatic editing [110]. While waiting for more objective criteria to identify a universally accepted editing procedure, we recommend that, if there are sufficient measurements, editing is not necessary for calculating average 24-h, daytime and night-time values, and that only grossly incorrect readings should be deleted from recordings (Table 6). The standard deviation is strongly affected by outliers, and its value should be related to the particular editing procedure applied to the recording.

\section{Agreement on definition of normality for ABPM}

As with CBPM, normal ranges for ABPM have been the subject of much debate over the years. Currently, an average daytime ABPM of less than $135 \mathrm{mmHg}$ systolic and $85 \mathrm{mmHg}$ diastolic is generally considered normal $[78,81]$, but even lower values are being advocated [89] particularly in high-risk groups, such as diabetic patients, in whom values less than 130/ $80 \mathrm{mmHg}$ are considered optimal [75-83,112-118]. The values shown in Table 6 are commonly used, although they are regarded as conservative by some. A prospective study from Japan has demonstrated that ABPM has a prognostic value, and that reference levels can be derived from long-term cardiovascular mortality [99]. The evidence coming from cross-sectional and current longitudinal studies supports lower levels of normality for ABPM, although the levels to be targeted by treatment remain to be established.
Table 6 Recommended levels of normality for ambulatory blood pressure measurements in adults

\begin{tabular}{llll} 
& Optimal & Normal & Abnormal \\
\hline Awake & $<130 / 80$ & $<135 / 85$ & $>140 / 90$ \\
Asleep & $<115 / 65$ & $<120 / 70$ & $>125 / 65$
\end{tabular}

Support for the normal and abnormal demarcation values is based on firm evidence from a number of studies; evidence is not yet available to make recommendations for the intermediate pressure ranges between 'normal' and 'abnormal', or for recommendations lower than those given. It must be emphasized that these values are only a guide to 'normality' and that lower 'optimal' values may be more appropriate in patients whose total risk-factor profile is high, and in whom there is concomitant disease, such as diabetes mellitus.

\section{Limitations of ABPM}

The technique of ABPM has many advantages over other available techniques but it has its limitations, which include:

- the provision of intermittent measurements during which the individual is sedentary rather than 'ambulatory'

- the fact that the technique makes demands on the individual

- that it may cause discomfort, and is more expensive than conventional measurement.

In addition, repeated ABPMs are often not welcomed by patients. These issues have been well addressed in recent publications $[119,120]$.

\section{Clinical indications for ABPM}

Ambulatory blood pressure monitoring provides a large number of blood pressure measurements over a period of time - usually the 24-h period - which can be plotted to give a profile of blood pressure behaviour. Although, in practice, the average daytime or nighttime values for ABPM are used to govern decisions, the clinical use of ABPM has enabled a number of phenomena in hypertension to be more clearly identified than is possible with other methods of blood pressure measurement [90] (Fig. 1 and Table 4). ABPM can benefit patients with hypertension who can be classified in the categories listed in Table 7.

\section{Patients with suspected white-coat hypertension}

The importance of white-coat hypertension rests on a curious haemodynamic phenomenon, which has quite profound clinical relevance: some patients - let us call them people, because they may not be ill - who appear to have hypertension when their blood pressure is measured by the traditional Riva-Rocci/Korotkoff method, have normal blood pressures when ABPM is used to record their blood pressures away from the medical environment. Put another way, conventional blood pressure measurement is misleading in people with white-coat hypertension and if decisions are based on 
Table 7 Possible clinical indications for ambulatory blood pressure measurement

- Suspected white-coat hypertension

- Suspected nocturnal hypertension

- To establish dipper status

- Resistant hypertension

- Elderly patient

- As a guide to antihypertensive drug treatment

- Type 1 diabetes

- Hypertension of pregnancy

- Evaluation of hypotension

- Autonomic failure

these measurements, inappropriate diagnosis and treatment may result. Because of the limited association of white-coat hypertension with the alerting reaction to the presence of a physician, it has been suggested that the alternative term 'isolated office hypertension' should be used $[19,121]$.

The CMS in the USA has recently approved ABPM for reimbursement, but only for 'patients with suspected white-coat hypertension' in whom the CMS believes the information deriving from the technique 'is necessary in order to determine the appropriate management of the patient' [84]. Faced with the dilemma of being unable any longer to deny the evidence showing the value of $\mathrm{ABPM}$ on the one hand, and the need to prevent indiscriminate use of the technique on the other, the CMS has therefore selected white-coat hypertension as the only indication for ABPM that is approved for reimbursement [84]. This decision, which is likely to change the clinical management of hypertension in the USA, makes white-coat hypertension a condition of major importance. The decision by the CMS begs the question as to how the practising physician can select patients with white-coat hypertension. It might, indeed, be argued that all patients with an increased CBPM are candidates for ABPM. Unfortunately, the reimbursement rate that has been recommended by the CMS in the USA is so low as to have little effect in encouraging the widespread use of the technique. The most popular definition of whitecoat hypertension requires a blood pressure measured by conventional techniques in the office, clinic or surgery to be greater than $140 / 90 \mathrm{mmHg}$ on at least three occasions, with normal ABPM measurements throughout the 24-h period, except perhaps during the first $1 \mathrm{~h}$ of the 24-h recording, when the patient is under the pressor influence of the medical environment while having the monitor fitted [88].

White-coat hypertension is common, but the prevalence depends, of course, on how the condition is defined. The prevalence has been variably described as comprising $10-15 \%$ of clinic referrals for ABPM, with reports for prevalence in the population of around $10 \%$ $[25,122]$.
The clinical importance of white-coat hypertension centres on the argument as to whether or not it carries an entirely benign prognosis. If it carries little or no risk, then establishing the diagnosis has significant implications, not just for those newly referred individuals in whom the diagnosis is suspected, and who can be reassured, but also for a large proportion of patients who have been labelled as 'hypertensive' with conventional measurement, and from whom the burden of unnecessary drug therapy may often be lifted, at least temporarily. The clinical significance of white-coat hypertension has become more clear from a growing mass of data, including some event-based cohort studies [123-125], which suggest that individuals with increased blood pressure on CBPM, who have normal average daytime pressures on ABPM, have a risk of major cardiovascular events comparable to that of clinically normotensive individuals and less than that of individuals with increased daytime pressures. Conversely, some studies have suggested that patients with white-coat hypertension may be at increased risk, albeit a smaller risk than patients with sustained hypertension [88,122,126]. Evidence to date does not therefore permit a conclusive statement on this issue but, clearly, if patients with white-coat hypertension are at risk, the risk is very much smaller than that for patients with sustained hypertension.

Several hypertension guidelines [85-87] stipulate that suspected white-coat hypertension is an indication for ABPM. However, none of the guidelines elaborate as to how the practising physician may 'suspect' white-coat hypertension, and in fairness it has to be admitted that data allowing an estimate of the probability of whitecoat hypertension according to the clinical characteristics of individuals are very scarce (Table 6). An analysis of data from a number of studies [25,127-129] indicate that, in untreated persons with essential hypertension, the probability of white-coat hypertension increases in those with:

(a) office systolic blood pressure $140-159 \mathrm{mmHg}$ or diastolic blood pressure 90-99 $\mathrm{mmHg}$

(b) female sex

(c) non-smokers

(d) hypertension of recent onset

(e) limited number of blood pressure measurements in the office

(f) normal left ventricular mass.

No one group seems to be exempt from white-coat hypertension: it may affect the young, the elderly, normotensive and hypertensive individuals, and pregnant women. The consequences of failing to identify the condition are considerable. Young (and, indeed, not-so-young) people may be penalized for insurance and pension policies, and for employment. Life-long 
treatment may be prescribed unnecessarily, and if antihypertensive medication is given to people whose 24-h pressures are normal, they may be made unwell by the adverse effects of medication. In the elderly, in whom white-coat hypertension is common, the inappropriate use of drugs may have serious debilitating consequences. It is recommended that people with white-coat hypertension should have the diagnosis confirmed in 3-6 months and be followed at yearly intervals with $\mathrm{ABPM}$ in order to detect if and when sustained hypertension occurs [83].

\section{White-coat effect}

White-coat hypertension must be distinguished from the 'white-coat effect', which is the term used to describe the increase in pressure that occurs in the medical environment regardless of the daytime ABPM. In other words, the term indicates the phenomenon, found in most hypertensive patients, whereby CBPM is usually greater than the average daytime ABPM, which is nonetheless increased above normal. The importance of the phenomenon is that patients diagnosed as having severe hypertension by CBPM may have only moderate or mild hypertension on ABPM, because of a marked white-coat effect. Although the white-coat effect may contribute to white-coat hypertension, there seems to be no predictive association between the two entities [130]. The term 'white-coat effect' has also been used by some authors $[82,131]$ to categorize individuals with an unusually large difference between office and ambulatory readings, regardless of the actual level of either. According to this line of thinking, a clinically significant white-coat effect has been defined as a CBPM exceeding mean daytime ABPM by at least $20 \mathrm{mmHg}$ systolic or $10 \mathrm{mmHg}$ diastolic, or both [82]. Such a large whitecoat effect has been found in as many as $73 \%$ of treated hypertensive individuals [131], and it may occur more frequently in women than in men [132].

\section{Masked hypertension (reverse white-coat hypertension)}

Recently, a group of patients have been identified in whom CBPM is normal but ABPM is increased $[133,134]$. This phenomenon, which is more common in the elderly, has previously been given the awkward titles of 'reverse white-coat hypertension' or 'white-coat normotension', and now Pickering and his colleagues [134] have proposed the more sensible term 'masked hypertension', to denote increased blood pressure that is hidden until ABPM is performed. If, as has already been shown, ABPM gives a better classification of risk than CBPM, then it follows that these people should be regarded as being genuinely hypertensive. The problem for clinical practice is how to identify and manage these patients, who it is estimated may number as many as 10 million people in the USA [135]. The phenomenon might be suspected in individuals who have had an increased CBPM at some time, in young persons with normal or normal-high CBPM who have early left ventricular hypertrophy, in individuals with a family history of hypertension in two parents, patients with multiple risks for cardiovascular disease, and perhaps diabetic patients.

\section{Resistant hypertension}

Combined systolic and diastolic hypertension is the most common form of hypertension, and ABPM allows for a more detailed assessment of the severity of increase in systolic and diastolic pressures, and the duration of the increase throughout the 24-h period $[93,134]$. Moreover, the degree of white-coat effect can be quantified. In patients with resistant hypertension defined as a CBPM consistently greater than 140/ $90 \mathrm{mmHg}$ in spite of treatment with three antihypertensive drugs, ABPM may indicate that the apparent lack of response is attributable, in fact, to the whitecoat phenomenon. Moreover, greater values of ABPM result in a worse prognosis in patients with refractory hypertension, supporting the recommendation that $\mathrm{ABPM}$ is useful in stratifying the cardiovascular risk in patients with refractory hypertension [93].

\section{Elderly patients in whom treatment is being considered}

A number of patterns of ABPM may be found in elderly individuals. The results of the ABPM substudy of the Systolic Hypertension in Europe Trial showed that systolic blood pressure measured conventionally in the elderly may average $20 \mathrm{mmHg}$ more than daytime ABPM [136], thereby leading to inevitable overestimation of isolated systolic hypertension in the elderly and probable excessive treatment of the condition. Moreover, results from this study also show that systolic ABPM was a significant predictor of cardiovascular risk over and above conventional systolic blood pressure [136]. A number of ABPM patterns are found in the elderly, among which are hypotensive states resulting from baroreceptor or autonomic failure. As the elderly can be particularly susceptible to the adverse effects of blood pressure-lowering drugs, identification of hypotension becomes particularly important [37], although its management may present a considerable therapeutic challenge.

\section{Suspected nocturnal hypertension}

Ambulatory blood pressure measurement is the only non-invasive blood pressure measuring technique that permits measurement of blood pressure during sleep. The relevance of nocturnal hypertension has been controversial, but recent evidence has shown that a non-dipping nocturnal pattern is a strong independent risk factor for cardiovascular mortality [89,91,92,137140]. In a recent study, [139], Ohkubo and his colleagues extended their previous preliminary findings [140] in 1542 inhabitants of Ohasama, Japan, by increasing the mean follow-up from 4.1 years to 9.2 years. There 
was a linear and inverse relationship between cardiovascular mortality and the nocturnal decline in blood pressure, which was independent of the overall blood pressure load during $24 \mathrm{~h}$ and other cardiovascular risk factors. Overall, each $5 \%$ increment in the systolic or diastolic night: day ratio was associated with a $20 \%$ increase in the risk of cardiovascular death, even when 24-h ABPM was within the normotensive range $(<135 /$ $80 \mathrm{mmHg}$ ). It has also been shown that absence of nocturnal 'dipping' of blood pressure to lower values than during the day is associated with target-organ involvement, and may be a useful (although nonspecific) clue as to the presence of secondary hypertension [83]. Nocturnal hypertension may be suspected on the basis of increased SBPM at home during the evening (although awake blood pressures correlate very poorly with nocturnal blood pressure), or by evidence of target-organ damage in excess of what might be expected from CBPM readings. When non-dipping (or extreme dipping) appears likely on ABPM, the recording should be repeated to confirm the diagnosis, as the reproducibility of nocturnal blood pressure is poor.

\section{Pregnancy}

As in the non-pregnant state, in pregnancy the main use for ABPM is the identification of white-coat hypertension, which may occur in nearly $30 \%$ of pregnant women [141]. Its recognition is important, so that pregnant women are not admitted to hospital or given antihypertensive drugs unnecessarily or excessively. Normal values for ABPM in the pregnant population are available, and the changes in pressure that occur during the trimesters of pregnancy and in the postpartum period have been defined (Table 8) [142]. The evidence that ABPM may predict pre-eclamptic toxaemia is not yet conclusive [143,144]. However ABPM correlates better with proteinuria than does CBPM [145], it is a better predictor of hypertensive complications [146], and women diagnosed by the technique as having hypertension have infants with lower birth weight than normotensive women [147]. Moreover, women with white-coat hypertension tend to have more caesarean sections than normotensive women, suggesting that, if $\mathrm{ABPM}$ rather than CBPM was used to measure blood pressure, caesarean delivery might be avoided [141].

\section{Diabetes}

In individuals with type 1 diabetes, a blunted or absent decrease in blood pressure from day to night is a predictor for increased risk of serious cardiovascular complications [148]. An increase in systolic ABPM during the night may antedate the development of microalbuminuria in young patients with type 1 diabetes, and the magnitude of the nocturnal dip in blood pressure predicts the development of microalbuminuria [149]. These data suggest that use of ABPM in patients
Table 8 Ambulatory blood pressure values during the trimesters of pregnancy

\begin{tabular}{|c|c|c|c|c|}
\hline \multirow[b]{2}{*}{$\mathrm{BP}(\mathrm{mmHg})$} & \multicolumn{4}{|c|}{ Gestation (weeks) } \\
\hline & $9-16$ & $18-24$ & $26-32$ & $33-40$ \\
\hline \multicolumn{5}{|l|}{ Daytime } \\
\hline SBP & $115 \pm 8$ & $115 \pm 8$ & $116 \pm 9$ & $119 \pm 9$ \\
\hline DBP & $70 \pm 7$ & $69 \pm 6$ & $70 \pm 7$ & $74 \pm 7$ \\
\hline \multicolumn{5}{|l|}{ Night-time } \\
\hline SBP & $100 \pm 7$ & $99 \pm 8$ & $101 \pm 8$ & $106 \pm 8$ \\
\hline DBP & $55 \pm 5$ & $54 \pm 6$ & $55 \pm 6$ & $58 \pm 7$ \\
\hline
\end{tabular}

Values are mean $\pm \mathrm{SD}$. BP, blood pressure; SBP, DBP, systolic and diastolic blood pressures. Data from O'Brien et al. [142].

with type 1 diabetes and absence of microalbuminuria may be valuable in directing therapeutic strategies to prevent renal disease at a very early stage.

Through carefully taken CBPM will identify most patients with type 2 diabetes in need of treatment, ABPM with identify a significant number of patients with 'masked hypertension' [150].

\section{Ambulatory hypotension}

Reference has already been made to the clinical use of ABPM in identifying hypotensive episodes in the elderly, but it may also be used in young patients in whom hypotension is suspected of causing symptoms [32]. In treated hypertensive patients, ABPM may also demonstrate drug-induced decreases in blood pressure that may have untoward effects in those with a compromised arterial circulation, such as individuals with coronary and cerebrovascular disease [151].

\section{Ambulatory blood pressure measurement and drug treatment}

The role of ABPM in guiding drug treatment is currently the subject of much research, and its place in this regard has not yet been fully established. However, recent reviews have highlighted the potential of 24-h ABPM in guiding the use of antihypertensive medication [152,153]. Furthermore, in a well-controlled study, adjustment of antihypertensive treatment on the basis of either ABPM or CBPM resulted in less intensive drug treatment in the ABPM group despite comparable blood pressure control in both groups and, importantly, patients in the ABPM group, who received less drug treatment, were not disadvantaged as judged by left ventricular mass on echocardiography [103]. Quite apart from this attribute, ABPM gives the prescribing doctor an assessment of the response to treatment that CBPM cannot provide: the efficacy of treatment without the white-coat effect can be ascertained, excessive drug effect and the occurrence of symptoms can be determined, and the duration of drug effect over the 24-h period and the consequences for blood pressure control of failing to take a drug as prescribed can be demon- 
strated [154]. The 24-h distribution of the blood pressure effect of antihypertensive drugs can also be quantified by applying specific mathematical indices to the analysis of ABPM recordings, such as the trough-topeak ratio and the smoothness index [155-157].

\section{Who should be re-monitored?}

Ambulatory blood pressure monitoring may cause inconvenience to patients, and it should be used, therefore, with discretion. The decision as to when to repeat ABPM is largely one of clinical judgement, which may be influenced by factors such as excessive blood pressure variability, an inappropriate response to treatment, an adverse risk-factor profile and the need for careful control of blood pressure, such as in hypertensive patients with diabetes mellitus, renal disease or severe cardiovascular disease.

In individuals with increased CBPM and normal ABPM - that is, white-coat hypertension - it is advisable to confirm the reproducibility of the diagnosis by repeating ABPM within 3-6 months, because more than $50 \%$ of those with white-coat hypertension may develop sustained hypertension within 6 months [158]. As a general rule, in individuals with confirmed white-coat hypertension and a normal risk factor profile it is usually unnecessary to repeat ABPM more frequently than annually, or every 2 years if the pattern appears to be established and consistent, as is often the case [77]. Conversely, if a patient with white-coat hypertension has a high-risk profile, ABPM every 6 months may be indicated, in order to detect the transition from the white-coat hypertension state to sustained hypertension and the need for antihypertensive medication. Alternatively, SBPM may be combined with ABPM to reduce the frequency of ABPM.

The frequency of repeat ABPM to evaluate the efficacy of antihypertensive medication will be dependent on the severity of the hypertension and the response to treatment. In patients with severe hypertension and evidence of target-organ damage, blood pressure reduction is urgent, and in the initial stages of treatment ABPM may be required monthly or sometimes more frequently as different drug combinations are introduced and dosages are altered. In patients with mild hypertension and no evidence of target-organ involvement, ABPM every 6 months may suffice to guide drug treatment.

Hypertensive patients with a marked white-coat reaction can be particularly difficult to manage because CBPM may be very misleading, and management must then be governed by ABPM. The frequency of repeat ABPM must be guided by the response to treatment, the stability of blood pressure control and the overall cardiovascular risk profile.
The frequency of ABPM in other indications for the technique will depend on the overall cardiovascular status of the patient and the overall response to management. In patients in whom the risk for cardiovascular complications is high, the frequency of repeat ABPM is more than justified by the need for tight control of blood pressure, whereas when the risk is low, less frequent $\mathrm{ABPM}$ is required, or the use of the technique can be combined with SBPM.

\section{Conclusion}

Ambulatory blood pressure measurement has been used in research for many years and is now mandatory in clinical pharmacological research and when new antihypertensive compounds are being assessed. After a long gestational period in research, ABPM has now become an indispensable technique in the management of hypertension. This being so, there is a need to encourage its use in general practice, rather than restricting its availability to specialist hospital centres, as has tended to be the case hitherto. However, diagnostic and management decisions based on the interpretation of ABPM patterns are more complex than has been the case with CBPM, and suitable educational processes must also be initiated. Standardization of data handling and presentation, the display of normal bands, the delineation of the windows of the $24 \mathrm{~h}$ profile and computer-generated reports as physician assistance are steps that should make the technique easier to use and interpret, so that its manifest advantages can be utilized to improve the management of hypertension, which remains so abysmally poor.

\section{Part IV. Self blood pressure measurement}

It has been recognized for more than 50 years that SBPM readings in the home are lower than those recorded by a doctor [74,159]. The discrepancy between pressures recorded in the home and the clinic, which has been confirmed repeatedly, is primarily a result of the white-coat effect, and is present regardless of whether patients or their relatives measure the blood pressure [160].

Self blood pressure measurement is popular among patients, as indicated by the huge sales of self-monitoring devices, but SBPM has not been accepted as readily in medical practice as home blood glucose monitoring in diabetes has been. There are a number of explanations for this:

- training patients to use SBPM using the auscultatory technique was troublesome and time-consuming, and not suitable for many individuals

- SBPM is subject to bias, whereby patients can record pressures of their own making

- SBPM may induce anxiety in some patients, who may take an obsessional interest in blood pressure 
- most automated devices available for SBPM have not been validated adequately, or have been shown to be inaccurate

- because of these limitations, data have been lacking to provide the evidence needed to ensure the place of SBPM in modern clinical practice.

However, the advent of accurate inexpensive automated devices that can provide a printout of blood pressure measurement with the time and date of measurement, or which allow storage of data for later analysis, plotting or electronic transmission, has removed some of these drawbacks, and there is now a renewed interest in SBPM. This revival of interest in an old methodology was recognized when experts from around the world gathered at the First International Consensus Conference on Self Blood Pressure Measurement, in Versailles in 1999, to discuss the evidence for and against the technique and to establish guidelines for its use in clinical medicine [161-168]. One of the recurring themes of the conference was the need for further research to determine the precise role of SBPM in practice. The discussion and recommendations that follow are based, with permission, on the deliberations of this conference.

The drafting of guidelines for SBPM comes at an important moment in the global approach to the management of hypertension, and particularly for the use of the various blood pressure measuring methods. In fact, the most recent international guidelines for the management of hypertension, while highlighting the need to stratify total cardiovascular risk accurately, have acknowledged the need to look to alternative methods of measurement, such as SBPM and ABPM, in order to quantify more precisely the contribution of hypertension to cardiovascular risk [85-87].

\section{General considerations Devices and validation}

The types of monitors available for SBPM include mercury-column sphygmomanometers, aneroid manometers and electronic semi-automatic or automatic devices. Although patients occasionally wish to use portable mercury-column sphygmomanometers, they are cumbersome, require technical instructions and training of the patient, and they contribute to environmental pollution with mercury [64-68]. Aneroid manometers, which have been widely used by patients, also require skill by the patient and training by the physician, and they can become inaccurate with use [57]. The availability of electronic devices has increased markedly during the past few years and they are popular with patients.

The sale of electronic blood pressure measuring devices designed for SBPM is not necessarily subject to any medical influence. Such devices can be bought by patients without discussion with their doctors, and can be advertised outside the pharmacy or medical distribution system without medical constraint. This freedom from medical control, coupled with a growing public desire to know more about health and illness, has resulted in the manufacture and marketing of a vast array of such devices, few of which have been evaluated according to the procedures considered necessary for blood pressure measuring equipment used in clinical practice. Therefore, in the interest of obtaining accurate SBPM measurements, the public should be advised to purchase only devices that have been independently validated (Table 9).

The automated devices available for SBPM all use the oscillometric technique. There are three categories available: devices that measure blood pressure on the upper arm, the wrist and the finger.

Finger devices. Devices that measure blood pressure at the finger are not recommended, because of the inaccuracies that occur because of measurement distortion with peripheral vasoconstriction, the alteration in blood pressure the more distal the site of recording, and the effect of limb position on blood pressure [2,8].

Wrist devices. Devices that measure blood pressure at the wrist are subject to the same last two problems as the finger devices, and although they are more accurate than finger measuring devices, there are strong reservations about the correct use of wrist devices. If the wrist is not held at heart level during measurement, inaccurate measurements will be obtained, and measurement is also influenced by flexion and hyperextension of the wrist. Additional studies have been advocated to explore the role of wrist measuring devices in special populations, such as obese or elderly individuals, in whom SBPM using the upper arm is more difficult to perform [169]. Because correct placement of the cuff at heart level is essential for accurate measurement with wrist devices, some devices have an inbuilt position sensor to indicate the correct position [170,171]. Despite their many limitations, automatic wrist monitors are popular among patients, because measurement is readily obtained without the need to remove clothing.

Upper arm devices. Monitors that measure blood pressure in the upper arm (brachial artery) have been shown

Table 9 Devices and validation

- Devices must have an EU certificate

- Devices should be validated using established protocols

- Arm measuring devices are the recommended choice

- Finger measuring devices are not recommended

- Wrist measuring devices can be unreliable

- Correct cuffs for individual arms should be used

- Manufacturers should provide an 'adjustable cuff' applicable to all adult arms 
to be the most reliable devices, both in clinical practice and in the major hypertension trials; therefore their use is recommended for SBPM. The recommendations that apply to blood pressure in general are applicable to these automated devices (see Part I). Appropriate cuff sizes should be available. It may not be possible to measure blood pressure with automated devices in patients with arrhythmias, and there are some patients in whom automated measurement is not possible without an obvious reason being evident. In view of the fact that the number of devices for SBPM on the market that have fulfilled independent validation criteria is small, the state of the market needs to be assessed regularly, with the results being easily accessible to prospective purchasers. In this regard, the ESH and BHS have established websites to provide updated lists of validated blood pressure measuring devices [10,11].

Choice of an appropriate device. Preference should be given to devices using a brachial cuff and offering the possibility to store, transmit or print measurements. Wrist instruments should be considered with caution. Manual devices based on the auscultatory method may be more appropriate for patients with arrhythmias (see Part II).

\section{User procedure}

The recommendations for SBPM do not vary in principle from those that apply for blood pressure measurement in general, but there are some points in need of emphasis:

- SBPM should be performed under medical supervision

- there should be a 5-min period of rest before measurement

- the cuff of the measuring device must be at the level of the heart on the arm with the highest blood pressure

- during diagnosis and initiation of treatment, duplicate SBPM measurements should be made in the morning and evening for 1 week, and for long-term observation this should be repeated for 1 week every 3 months, with the first day of measurements being discarded

- patients' diaries may be unreliable, and memoryequipped devices are preferred.

Use in primary care. At present, SBPM is performed mostly by patients on their own initiative, using devices bought on the free market, without medical control. Primary care physicians should see SBPM as a means of gaining further insight into blood pressure control and the effects of management strategies in motivated and informed patients who remain under medical supervision.
Frequency and timing of SBPM. The frequency of SBPM may vary according to the indication and the information that is being sought [165]. The difference between morning and evening values is greater in treated than in untreated individuals, and this is probably attributable to the effect of antihypertensive medication, reflecting perhaps the duration of blood pressure lowering [172]. Although no study has analysed the optimal timing and frequency of measurements, the following recommendations of the German Hypertension League are proposed [165]:

- Commencement phase: At the initiation of SBPM, there should be an initial 7-day measurement period with two SBPMs in the morning and in the evening at predefined times (0600-0900 h and 0600-2100 h). Measurements from the first day should be excluded from the statistical analysis, because these may represent a period of familiarization and anxiety with the technique, and often yield measurements that are not representative [173]. The average of these values is taken as reference for the follow-up period.

- Treatment phase: SBPM should be used exactly as in the commencement phase. The SBPM data during treatment should, as a matter of principle, be ascertained at trough - that is, before the drug intake. If treatment is changed, the averages of the SBPM values measured over 2 weeks are used to assess the treatment effect [165].

- Follow-up phase: SBPM should be performed on one day per week if hypertension is controlled. This frequency of measurement may be increased in patients in whom poor compliance to treatment, or uncontrolled hypertension is suspected.

The minimum of measurements performed in each period should be a total of 12 readings recorded during 7 working days. Depending on individual needs (e.g. poor compliance) or for pharmacological studies, more frequent measurements may be recommended [165].

\section{Factors influencing SBPM}

Observer prejudice. The unreliability of SBPM reported by patients themselves has been demonstrated by comparing the recorded pressures with those recorded secretly by an automatic data storage system [174]. Memory-equipped devices have the potential to reduce observer prejudice.

Training of patients. Doctors must themselves be conversant with the strengths and limitations of SBPM, be aware of the accuracy and reliability of the equipment being used by their patients, and be able to advise them on the state of the market for automated devices [175]. Training should focus on equipment, the procedure, interpretation of results, blood pressure variability, normal readings, the need for the calibration and mainte- 
nance of the equipment, and also hypertension and its treatment. Towards this end, nurses in primary care practices, who are most suited to training patients, may find the available CD-ROMs [22,23,176] and the BHS booklet useful for demonstration to patients anxious to know more about self measurement [19].

Seasonal variations. Blood pressure varies with the seasons, being higher in winter and lower in summer, and these variations should be considered in the interpretation of SBPM measurements in individual patients [177].

Patient disability. Few patients are unable to perform SBPM, but it may be unsuitable for patients with physical problems, or mental disabilities. SBPM is most suited to hypertensive patients who wish to contribute to their own management.

\section{Diagnostic thresholds}

The association between blood pressure and cardiovascular risk is continuous, without a threshold above which risk suddenly increases. However, clinical decisions must be based on diagnostic or operational thresholds. In this regard, there is an agreement that the thresholds currently applicable for conventional sphygmomanometry cannot be extrapolated to automated measurements. Different methodological approaches may be used for the determination of threshold values, the most satisfactory of which is to relate blood pressure thresholds to cardiovascular outcome, but there are limited data available for SBPM [178-180]. The recommended thresholds shown in Table 10 are derived from statistical considerations in a large population database comprising some 5422 normotensive and untreated hypertensive individuals [164,179]. The threshold of $135 / 85 \mathrm{mmHg}$ for SBPM is the same as that for mean daytime ABPM.

\section{Clinical indications}

The clinical applications of SBPM are only beginning to become apparent as the technique becomes more widely used and scientific data are gathered.

\section{White-coat hypertension}

CBPM is extremely variable over time, and the accuracy of the technique may be improved by increasing the number of visits, rather than the number of measurements at a given visit. SBPM has been proposed as a useful alternative to ABPM in the detection of white-

Table 10 Diagnostic thresholds for self blood pressure measurement (SBPM)

- Data from longitudinal studies limited

- Reference values are derived principally from statistical evaluation of databases

- $135 / 85 \mathrm{mmHg}$ may be considered as upper limit of normality

- SBPM needs to be further evaluated in prospective outcome studies coat hypertension [181], but the finding of a normal SBPM does not rule out the possibility that the blood pressure may be higher at other times of the day. The same magnitude of the white-coat effect and the same proportion of clinic reactors have been observed using SBPM and ABPM, but there was disagreement in the classification of a substantial number of patients. The CBPM-SBPM difference is often smaller than the difference between CBPM and average daytime ABPM [182-185]. SBPM, which is less costly and more convenient than ABPM, may be appropriate for the long-term follow-up of patients with white-coat hypertension.

\section{Guiding antihypertensive medication}

SBPM may have a role in assessing the response to antihypertensive drug treatment outside the medical environment and over time. Measuring blood pressure in the home environment under similar every-day conditions avoids the white-coat effect and allows a reduction in variability $[168,182,186]$. SBPM can improve the assessment of blood pressure measurement in hypertension management and in clinical trials. In fact, SBPM has been shown to be a sensitive method for detecting small changes in pressure. Moreover, the duration of action of an antihypertensive drug can be assessed. SBPM also has a potential role in trials of antihypertensive medication in which self measurements can be carried out a number of times each day over several weeks [186,187].

\section{The elderly}

The feasibility of using SBPM in elderly patients may be influenced by physical and intellectual limitations and by the complexity of use of the chosen device. Studies have shown that automated devices are more precise and easier to use than semi-automatic and manually operated equipment in elderly people.

\section{Pregnancy}

As in the general population, in pregnant women the blood pressures recorded by SBPM are lower than those obtained by CBPM. SBPM may be useful in diagnosing white-coat responders and monitoring the effect of antihypertensive medication. The use of data storage and electronic transmission of data may have a particular role for patients living at a distance from the maternity clinic. However, there are very few data on the normal values for SBPM in the different stages of pregnancy and on the use of the technique in pregnancy.

\section{Diabetes}

There is increasing evidence that stringent control of blood pressure reduces the cardiovascular and microvascular complications of diabetes, and SBPM may provide an additional means of ensuring that such 
control is achieved, although there are as yet no data to guide the use of SBPM in diabetic patients.

\section{Resistant hypertension}

In patients with apparently uncontrolled CBPM, the blood pressure may be adequately controlled when they are at home. It may be possible to identify at least some of these patients by SBPM, although ABPM is the preferred technique. In the evaluation of patients with resistant hypertension but no signs of target-organ damage, the first step might be to use SBPM, and if the blood pressures are low, ABPM may then be indicated to confirm the degree of control.

\section{Improving compliance to treatment}

One of the most important causes of refractory hypertension is poor compliance or adherence to treatment. SBPM may provide patients with an understanding of increased blood pressure and its response to treatment, thereby improving adherence to treatment $[188,189]$.

\section{Predicting outcome}

SBPM may offer some advantage over CBPM in predicting cardiovascular outcome in hypertension [175], but data are extremely limited and the results of current trials must be awaited. However, the results of cross-sectional studies have shown that the degree of left ventricular hypertrophy determined by electrocardiography and by echocardiography is more strongly correlated to SBPM than to CBPM [167,190,191].

\section{References}

1 Beevers G, Lip GHY, O'Brien E. ABC of references hypertension. Part I. Sphygmomanometry: factors common to all techniques. BMJ 2001; 322:981-985.

2 O'Brien E, Waeber B, Parati G, Staessen J, Myers MG. European Society of Hypertension recommendations on blood pressure measuring devices. BMJ 2001; 322:532-536.

3 Association for the Advancement of Medical Instrumentation. American National Standard for Electronic or Automated Sphygmomanometers. ANSI/AAMI SP10 - 1987. Arlington, VA: AAMI; 1987.

4 O'Brien E, Petrie J, Littler WA, Padfield PL, O'Malley K, Jamieson M, et al. British Hypertension Protocol: evaluation of automated and semiautomated blood pressure measuring devices with special reference to ambulatory systems. J Hypertens 1990; 8:607-619.

5 Association For The Advancement Of Medical Instrumentation. American National Standard for Electronic or Automated Sphygmomanometers: ANSI/AAMI SP10 - 1993. Arlington, VA: AAMI; 1993.

6 O'Brien E, Petrie J, Littler WA, de Swiet M, Padfield PL, Altman D, et al. The British Hypertension Society Protocol for the evaluation of blood pressure measuring devices. J Hypertens 1993; 11 (suppl 2): S43-S63.

7 O'Brien E, Pickering T, Asmar R, Myers M, Parati G, Staessen J, et al., on behalf of the Working Group on Blood Pressure Monitoring of the European Society of Hypertension. International protocol for validation of blood pressure measuring devices in adults. Blood Press Monit 2002; 7:3-17.

8 O'Brien E, Atkins N, Staessen J. State of the market: a review of ambulatory blood pressure monitoring devices. Hypertension 1995; 26:835-842.

9 O'Brien E. State of the market in 2002 for blood pressure measuring devices. Blood Press Monit 2001; 6:171-176.

10 European Society of Hypertension: http://www.eshonline.org/ newsletter/2002/Newsletter_nr12.pdf

11 British Hypertension Society: http://www.hyp.ac.uk/bhs/bp_monitors/ automatic.htm
12 European Commission for Standardisation. European Standard EN 1060-1 (British Standard BSSEN 1060-1:1996). Specification for noninvasive sphygmomanometers. Part I. General requirements. 1995. Brussels: European Commission for Standardisation; 1995

13 European Commission for Standardisation. European Standard EN 1060-2 (British Standard BSSEN 1060-2:1996). Specification for noninvasive sphygmomanometers. Part 2. Supplementary requirements for mechanical sphygmomanometers. 1995. Brussels: European Commission for Standardisation; 1995.

14 European Committee for Standardization. European Standard EN 10603 1997 (British Standard BSEN 1060-3: 1997). Specification for noninvasive sphygmomanometers. Part 3. Supplementary requirements for electro-mechanical blood pressure measuring systems. Brussels: European Committee for Standardization; 1997.

15 Normenausschuß Feinmechanik und Optik (NaFuO) im DIN Deutsches Institut für Normierung eV. Non-invasive sphygmomanometers - clinical investigation. Berlin: Beuth Verlag; 1996.

16 Mancia G, Bertinieri G, Grassi G, Parati G, Pomidossi G, Ferrari A, et al. Effects of blood pressure measurement by the doctor on patient's blood pressure and heart rate. Lancet 1983; ii:695-698.

17 Mancia G, Ferrari A, Gregorini L, Parati G, Pomidossi G, Bertinieri G, et al. Blood pressure and heart rate variabilities in normotensive and hypertensive human beings. Circ Res 1983; 53:96-104.

18 Parati G, Ulian L, Santucciu C, Omboni S, Mancia G. Blood pressure variability, cardiovascular risk and antihypertensive treatment. J Hypertens 1995; 13 (suppl 4): S27-S34.

19 Parati G, Ulian L, Santucciu C, Omboni S, Mancia G. Difference between clinic and daytime blood pressure is not a measure of the white coat effect. Hypertension 1998; 31:1185-1189.

20 O'Brien E, Petrie J, Littler WA, de Swiet M, Padfield PD, Dillon MJ, et al. Blood pressure measurement: recommendations of the British Hypertension Society, 3rd edn. London: BMJ Publishing Group; 1997.

21 Beevers G, Lip GYH, O'Brien E. ABC of hypertension, 4th edn. London: BMJ Books; 2001.

22 British Hypertension Society. Blood pressure measurement [CD-ROM] 1998. Available from BMJ Books, BMA House, Tavistock Square, London WC1H 9JR.

23 Société Française d'Hypertension Artérielle. La prise de la pression artérielle au cabinet medical [CD-ROM] 1998. Available from Société Française d'Hypertension Artérielle.

24 Parati G, Ulian G, Sampieri L, Palatini P, Villani A, Vanasia A, et al., on behalf of the Study on Ambulatory Monitoring of Blood Pressure and Lisinopril Evaluation (SAMPLE) Study Group. Attenuation of the 'white coat effect' by antihypertensive treatment and its relationship to regression of target organ damage. Hypertension 2000; 35:614-620.

25 Pickering TG, James GD, Boddie C, Harshfield GA, Blank S, Laragh JH. How common is white coat hypertension? JAMA 1988; 259:225-228.

26 Silverberg DS, Shemesh E, Jaina A. The unsupported arm: a cause of falsely raised blood pressure readings. BMJ 1977; 2:1331.

27 O'Brien E, O'Malley K. The ABC of blood pressure measurement: the sphygmomanometer. BMJ 1979; 2:851-853.

28 Lane D, Beevers M, Barnes N, Bourne J, John A, Malins S, et al. Interarm differences in blood pressure: when are they clinically significant? $J$ Hypertens 2002; 20:1089-1095.

29 Gosse P. Blood pressure should be measured in both arms on the first consultation. J Hypertens 2002; 20:1045-1046.

30 O'Brien E. A century of confusion: which bladder for accurate blood pressure measurement? [review] J Hum Hypertens 1996; 10:565-572.

31 Pickering T. Ambulatory monitoring and blood pressure variability, Part I. London: Science Press; 1990. p. 2.8.

32 Perloff D, Grim C, Flack J, Frohlich ED, Hill M, McDonald M, et al. Human blood pressure determination by sphygmomanometry. Circulation 1993; 88:2460-2470.

33 De Swiet M, Dillon MJ, Little W, O'Brien E, Padfield PL, Petrie JC. Measurement of blood pressure in children. Recommendations of a working party of the British Hypertension Society. BMJ 1989; 299:497.

34 National High Blood Pressure Education Group on Hypertension Control in Children and Adolescents. Update on the 1987 Task Force report on high blood pressure in children and adolescents: a working group report from the National High Blood Pressure Education Program. Pediatrics 1996; 98:649-658.

35 Staessen JA, Fagard R, Thijs L, Celis H, Arabidze GG, Birkenhager $\mathrm{WH}$, et al., for the Systolic Hypertension in Europe (Syst-Eur) Trial Investigators. Randomised double-blind comparison of placebo and active treatment for older patients with isolated systolic hypertension. Lancet 1997; 350:757-764.

36 O'Brien E, O'Malley K. Blood pressure measurement in the elderly with special reference to ambulatory blood pressure measurement. In: 
Leonetti G, Cuspedi C (editors): Hypertension in the elderly. Amsterdam: Kluwer; 1994. pp. 13-25.

37 Owens P, O'Brien ET. Hypotension: a forgotten Illness? Blood Press Monit 1996; 2:3-14.

38 Vanhanen H, Thijs L, Birkenhager W, Tilvis R, Sarti C, Tuomilehto J, et al., on behalf of the Syst-Eur Investigators. Associations of orthostatic blood pressure fall in older patients with isolated systolic hypertension. $J$ Hypertens 1996; 14:943-949.

39 Spence JD, Sibbald WJ, Cape RD. Pseudohypertension in the elderly. Clin Sci Mol Biol 1978; 55:399S-402S.

40 O'Callaghan WG, Fitzgerald D, O'Malley K, O'Brien E. Accuracy of indirect blood pressure measurement in the elderly. BMJ 1983; 286:1545-1546.

41 Staessen J, Fagard R, Amery A. The relationship between body weight and blood pressure. J Hum Hypertens 1988; 2:207-217.

42 Stewart MJ, Gough K, Padfield PL. The accuracy of automated blood pressure measuring devices in patients with controlled atrial fibrillation. $J$ Hypertens 1995; 13:297-300.

43 Shennan AH, Halligan AWF. Measuring blood pressure in normal and hypertensive pregnancy. Baillière's Clin Obstet Gynaecol 1999; 13:1-26.

44 Shennan A, Gupta M, Halligan A, Taylor DJ, de Swiet M. Lack of reproducibility in pregnancy of Korotkoff phase IV as measured by sphygmomanometry. Lancet 1996; 347:139-142.

45 de Swiet M. K5 rather than K4 for diastolic blood pressure measurement in pregnancy. Hypertens Pregnancy 1999; 18:iii-v.

46 Fletcher GF, Balady GJ, Amsterdam EA, Chaitman B, Eckel R, Fleg J, et $\mathrm{al}$. Exercise standards for testing and training: a statement for healthcare professionals from the American Heart Association. Circulation $2001 ; 104: 1694-1740$

47 Weisser B, Hartrumpf T, Mengden T, Glänzer K, Vetter H. Exercise blood pressure in the elderly - proposal for normal values in view of the 1999 WHO blood pressure classification. Dtsch Med Wochenschr 2001; 126 (suppl 3): S159.

48 Manolio TA, Burke GL, Savage PJ, Sidney S, Gardin JM, Oberman A. Exercise blood pressure response and 5-year risk of elevated blood pressure in a cohort of young adults: the CARDIA study. Am J Hypertens 1994; 7:234-241.

49 Matthews CE, Pate RR, Jackson KL, Ward DS, Macera CA, Kohl HW, Blair SN. Exaggerated blood pressure response to dynamic exercise and risk of future hypertension. J Clin Epidemio/ 1998; 51:29-35.

50 Singh JP, Larson MG, Manolio TA, O'Donnell CJ, Lauer M, Evans JC, Levy D. Blood pressure response during treadmill testing as a risk factor for new-onset hypertension: the Framingham heart study. Circulation 1999; 99:1831-1836.

51 Mundal R, Kjeldsen SE, Sandvik L, Erikssen G, Thaulow E, Erikssen J. Exercise blood pressure predicts mortality from myocardial infarction. Hypertension 1996; 27:324-329.

52 McHam SA, Marwick TH, Pashkow FJ, Lauer MS, George M, Kauffman LH. Delayed systolic blood pressure recovery after graded exercise: an independent correlate of angiographic coronary disease. J Am Coll Cardiol 1999; 34:754-759.

53 Gottdiener JS, Brown J, Zoltick J, Fletcher RD. Left ventricular hypertrophy in men with normal blood pressure: relation to exaggerated blood pressure response to exercise. Ann Intern Med 1990; 112: $161-166$.

54 Ren JF, Hakki AH, Kotler MN, Iskandrian AS. Exercise systolic blood pressure: a powerful determinant of increased left ventricular mass inpatients with hypertension. J Am Coll Cardiol 1985; 5 1224-1231.

55 Kjeldsen SE, Mundal R, Sandvik L, Erikssen G, Thaulow E, Erikssen J. Supine and exercise systolic blood pressure predict cardiovascular death in middle-aged men. J Hypertens 2001; 19:1343-1348.

56 Filipovsky J, Ducimetiere P, Safar ME. Prognostic significance of exercise blood pressure and heart rate in middle-aged men. Hypertension 1992; 20:333-339.

57 Beevers G, Lip GHY, O'Brien E. ABC of hypertension. Part II. Conventional sphygmomanometry: technique of auscultatory blood pressure measurement. BMJ 2001; 322:1043-1047.

58 Rose G. Standardisation of observers in blood pressure measurement. Lancet 1965; 1:673-674.

59 Keary L, Atkins N, Molloy E, Mee F, O'Brien E. Terminal digit preference and heaping in blood pressure measurement. J Hum Hypertens 1998; 12:787-788.

60 O'Brien E, Mee F, Atkins N, O'Malley K, Tan S. Training and assessment of observers for blood pressure measurement in hypertension research. J Hum Hypertens 1991; 5:7-10.

61 Fitzgerald D, O'Callaghan W, O'Malley K, O'Brien E. Inaccuracy of the
London School of Hygiene sphygmomanometer. BMJ 1982; 284: $18-19$

62 Conroy R, O'Brien E, O'Malley K, Atkins N. Measurement error in the Hawksley random zero sphygmomanometer: what damage has been done and what can we learn? BMJ 1993; 306:1319-1322.

63 Brown WCB, Kennedy S, Inglis GC, Murray LS, Lever AF. Mechanisms by which the Hawksley random zero sphygmomanometer underestimates blood pressure and produces a non-random distribution of RZ values. J Hum Hypertens 1997; 11:75-93.

64 O'Brien E. Replacing the mercury sphygmomanometer. BMJ 2000; 320:815-816.

65 O'Brien E. Ave atque vale: the centenary of clinical sphygmomanometry. Lancet 1996; 348:1569-1570.

66 O'Brien E. Will mercury manometers soon be obsolete? J Hum Hypertens 1995; 9:933-934.

67 O'Brien E. Consequences of banning mercury and the cuff controversy. Blood Press Monit 2000; 5:33-34.

68 O'Brien E. Will the millimetre of mercury be replaced by the kilopascal? $J$ Hypertens 1998; 16:259-261.

69 Burke MJ, Towers HM, O'Malley K, Fitzgerald D, O'Brien E. Sphygmomanometers in hospitals and family practice: problems and recommendations. BMJ 1982; 285:469-471.

70 Mion D, Pierin AMG. How accurate are sphygmomanometers? J Hum Hypertens $1998 ; 12: 245-248$.

71 Pickering T. What will replace the mercury sphygmomanometer? Blood Press Monit 2003; 8:23-25.

72 Sever PS, Dah1 off B, Poulter NP, Wedel H, Beevers G, Caulfield M, et al. Rationale, design, methods and baseline demography of participants of the Anglo-Scandinavian Cardiac Outcomes Trial. J Hypertens 2001:19:1139-1147

73 O'Brien E, Mee F, Atkins N, Thomas M. Evaluation of three devices for self-measurement of blood pressure: according to the revised British Hypertension Society Protocol: the Omron HEM-705CP, Phillips HP5332, and Nissei DS-175. Blood Press Monit 1996; 1:55-61.

74 O'Brien E, Fitzgerald D. The history of blood pressure measurement. $J$ Hum Hypertens 1994; 8:73-84.

75 Pickering T, O'Brien E, O'Malley K. Second international consensus meeting on twenty-four-hour ambulatory blood pressure measurement: consensus and conclusions. J Hypertens 1991; 9 (suppl 8):S2-S6.

76 Mancia G, Di Rienzo M, Parati G. Ambulatory blood pressure monitoring: use in hypertension research and clinical practice. Hypertension 1993; 21:500-524.

77 O'Brien E, Beevers G, Lip GHY. ABC of hypertension. Part III. Automated sphygmomanometry: ambulatory blood pressure measurement. BMJ 2001; 322:1110-1114.

78 Staessen JA, O'Brien ET, Thijs L, Fagard R. Modern approaches to blood pressure measurement. Occup Environ Med 2000; 57:510-520.

79 O'Brien E. Ambulatory blood pressure monitoring in the management of hypertension. Heart 2003; (in press).

80 Parati G, Bosi S, Castellano M, Cristofari M, Di Rienzo M, Germano G, et al. Guidelines for 24 hour non-invasive ambulatory blood pressure monitoring. Report from a working group of the Italian Society of Hypertension. High Blood Press 1995; 4:168-174.

81 Pickering $\mathrm{T}$, for the American Society of Hypertension Ad Hoc Panel. Recommendations for the use of home (self) and ambulatory blood pressure monitoring. Am J Hypertens 1996; 9:1-11.

82 Myers MG, Haynes RB, Rabkin SW. Canadian Hypertension Society guidelines for ambulatory blood pressure monitoring. Am J Hypertens 1999; 12:1149-1157.

83 O'Brien E, Coats A, Owens P, Petrie J, Padheld P, Littler WA, et al. Use and interpretation of ambulatory blood pressure monitoring: recommendations of the British Hypertension Society. BMJ 2000; 320: $1128-1134$.

84 CMS. Centers for Medicare \& Medicaid Services. Medicare coverage policy - decisions. ABPM Monitoring (\#CAG-00067N). 2001; http:// www.hcfa.gov/coverage/8b3-ff.htm

85 Guidelines Subcommittee of the World Health Organisation-International Society of Hypertension (WHO-ISH). 1999 World Health Organisation-International Society of Hypertension Guidelines for the Management of Hypertension. J Hypertens 1999; 17:151-183.

86 Ramsay LE, Williams B, Johnston GD, MacGregor GA, Poston L, Potter $\mathrm{JF}$, et al. Guidelines for management of hypertension: report of the third working party of the British Hypertension Society. J Hum Hypertens 1999; 13:569-592.

87 The Joint National Committee of Prevention, Detection, Evaluation, and Treatment of High Blood Pressure. The sixth report of the Joint National Committee on Prevention, Detection, Evaluation and Treatment of High Blood Pressure. Arch Intern Med 1997; 157:2443-2446. 
88 Owens P, Atkins N, O'Brien E. Diagnosis of white coat hypertension by ambulatory blood pressure monitoring. Hypertension 1999; 34: 267-272.

89 O'Brien E, Sheridan J, O'Malley K. Dippers and non-dippers [letter]. Lancet 1988; ii:397.

90 Owens P, Lyons S, O'Brien E. Ambulatory blood pressure in the hypertensive population: patterns and prevalence of hypertensive subforms. J Hypertens 1998; 16:1735-1743.

91 Asmar RG, Brunel PC, Pannier BM, Lacolley PJ, Safar ME. Arterial distensibility and ambulatory blood pressure monitoring in essential hypertension. Am J Cardiol 1988; 61:1066-1070.

92 Cunha RS, Benetos A, Laurent S, Safar ME, Asmar RG. Distension capacity of the carotid artery and ambulatory blood pressure monitoring. Effects of age and hypertension. Am J Hypertens 1995; 8:343-352.

93 Redon J, Campos C, Narciso ML, Rodicio JL, Pascual JM, Ruilope LM. Prognostic value of ambulatory blood pressure: monitoring in refractory hypertension. A prospective study. Hypertension 1998; 31:712-718.

94 Verdecchia P, Porcellati C, Schillaci G, Borgioni C, Ciucci A, Battistelli $\mathrm{M}$, et al. Ambulatory blood pressure. An independent predictor of prognosis in essential hypertension. Hypertension 1994; 24:793-801.

95 Staessen J, Thijs L, Fagard R, O'Brien E, Clement D, deLeeuw P, et al., for the Systolic Hypertension in Europe Trial Investigators. Predicting cardiovascular risk using conventional vs ambulatory blood pressure in older patients with systolic hypertension. JAMA 1999; 282:539-546.

96 Verdecchia P. Prognostic value of ambulatory blood pressure: current evidence and clinical implications. Hypertension 2000; 35:844-881.

97 Kario K, Pickering TG, Matsuo T, Hoshide S, Schwartz JE, Shimada K. Stroke prognosis and abnormal nocturnal blood pressure falls in older hypertensives. Hypertension 2001; 38:852-857.

98 Mancia G, Sega R, Bravi C, De Vito G, Valagussa F, Cesana G, et al. Ambulatory blood pressure normality: results from the PAMELA study. $J$ Hypertens 1995; 13:1377-1390.

99 Ohkubo T, Imai Y, Tsuji I, Nagai K, Ito S, Satoh H, Hisamichi S. Reference values for 24-hour ambulatory blood pressure monitoring based on a prognostic criterion: the Ohasama Study. Hypertension 1998; 32:255-259.

100 Atkins N, O'Brien E. DABL97 - a computer program for the assessment of blood pressure, risk factors and cardiovascular target organ involvement in hypertension. J Hypertens 1998; 16 (suppl 2):S198.

101 Moser M. The cost of treating hypertension, can we keep it under control without compromising the level of care? Am J Hypertens 1998; 11:120S-127S.

102 O'Brien E. Ambulatory blood pressure monitoring in primary care: a comment. Br J Cardiol 2001; 8:531-535.

103 Staessen JA, Byttebier G, Buntinx F, Celis H, O'Brien ET, Fagard R, for the Ambulatory Blood Pressure Monitoring and Treatment of Hypertension Investigators. Antihypertensive treatment based on conventional or ambulatory blood pressure measurement. A randomized controlled trial. JAMA 1997; 278:1065-1072.

104 Waeber B, Schubiger JL, Wohler D, Nawrath T, Richter A, Brandt A, et al. What are the costs or savings ambulatory blood pressure monitoring can produce in the treatment of hypertension? In: Proceedings of the XXXIVth International Conference on Applied Econometrics; 1992; Munich. pp. 131-137.

105 Fagard R, Staessen J, Thijs L. Optimal definition of daytime and nighttime blood pressure. Blood Press Monit 1997; 2:315-321.

106 Parati G, Rizzoni D, Omboni S, Bernardi L, Mormino P, Di Rienzo M. The analysis of blood pressure and heart rate variability: methodological aspects and interpretation of results. High Blood Press 1995; 4: $186-203$.

107 Stergiou GS, Malakos JS, Zourbaki AS, Achimastos AD, Mountokalakis TD. Blood pressure during siesta: effect on 24-hour ambulatory blood pressure profiles analysis. J Hum Hypertens 1997; 11:125-131.

108 Pickering T, Pieper C. Methods of analysis of ambulatory blood pressure data. In: Pickering TG (editor): Ambulatory monitoring and blood pressure variability. London: Science Press; 1991. pp. $16.1-16.15$.

109 Berardi L, Chau NP, Chanudet X, Vilar J, Larroque P. Ambulatory blood pressure monitoring: a critical review of the current methods to handle outliers. J Hypertens 1992; 10:1243-1248.

110 Winnicki M, Canali C, Mormino P, Palatini P. Ambulatory blood pressure monitoring editing criteria. Is standardization needed? Am J Hypertens 1997; 10:419-427.

111 Casadei R, Parati G, Pomidossi G, Groppelli A, Trazzi S, Di Rienzo M, Mancia G. 24-Hour blood pressure monitoring: evaluation of Spacelabs 5300 monitor by comparison with intra-arterial recording in ambulant subjects. J Hypertens 1988; 6:797-803.

112 Staessen J, Fagard R, Lijnen P, Thijs L, van Hoof R, Amery A.
Ambulatory blood pressure monitoring in clinical trials. $J$ Hypertens 1991; 9:S13-S19.

113 O'Brien ET, Staessen J. Normotension and hypertension as defined by 24-hour ambulatory blood pressure monitoring. Blood Pressure 1995; 4:266-282.

114 O'Brien E, Owens P, Staessen J, Imai Y, Kawasaki T, Kuwajima I. What are the normal levels for ambulatory blood pressure measurement? Blood Press Monit 1998; 3:131-132.

115 Mancia G, O'Brien E, Imai Y, Redon J. Task Force II: ambulatory blood pressure monitoring in population studies. Blood Press Monit 1999; 4:295-301.

116 Mancia G, Sega R, Grassi G, Cesana G, Zanchetti A. Defining ambulatory and home blood pressure variability. Further considerations from PAMELA study. J Hyperens 2001; 19:995-999.

117 Verdecchia P. Reference values for ABPM and self-measured blood pressure based on prospective outcome data. Blood Press Monit 2001; 6:323-328

118 Verdecchia P, Staessen JA, White WB, Imai Y, O'Brien ET. Properly defining white coat hypertension. Eur Heart J 2002; 23:106-109.

119 Parati GF, Mancia G. Ambulatory blood pressure monitoring in clinical practice. J Hypertens 2002; 20:1925-1927.

120 Palatini P. Too much of a good thing? A critique of overemphasis on the use of ambulatory blood pressure monitoring in clinical practice. J Hypertens 2002; 20:1917-1923.

121 Parati G, Redon J. Direct and surrogate measures of the white-coat effect: methodological aspects and clinical relevance. J Hypertens 2000; 18: 379-382.

122 Sega R, Trocino G, Lanzarotti A, Carugo S, Cesana G, Schiavina R, et al. Alterations in cardiac structure in patients with isolated office, ambulatory or home hypertension. Data from the PAMELA study. Circulation 2001; 104:1385-1392.

123 Verdecchia P, Schillaci G, Borgioni C, Ciucci A, Porcellati C. Whitecoat hypertension. Lancet 1996; 348:1444-1445.

124 Fagard RH, Staessen JA, Thijs L, Gasowski J, Bulpitt CJ, Clement D, et al. Response to antihypertensive therapy in older patients with sustained and nonsustained systolic hypertension. Systolic Hypertension in Europe (Syst-Eur) Trial Investigators. Circulation 2000; 102:1139-1144.

125 Kario K, Shimada K, Schwartz JE, Matsuo T, Hoshide S, Pickering TG. Silent and clinically overt stroke in older Japanese subjects with whitecoat and sustained hypertension. J Am Coll Cardiol 2001; 38:238-245.

126 Owens PE, Lyons SP, Rodriguez SA, O'Brien ET. Is elevation of clinic blood pressure in patients with white coat hypertension who have normal ABPM associated with target organ changes? J Hum Hypertens $1998 ; 12: 743-748$

127 Staessen JA, O'Brien ET, Atkins N, Amery AK, on behalf of the Ad-Hoc Working Group. Short report: ABPM in normotensive compared with hypertensive subjects. J Hypertens 1993; 11:1289-1297.

128 Sorof JM, Poffenbarger T, Franco K, Portman R. Evaluation of white coat hypertension in children: importance of the definitions of normal ABPM and the severity of casual hypertension. Am J Hypertens 2001; $14: 855-860$.

129 Verdecchia P, Palatini P, Schillaci G, Mormino P, Porcellati C, Pessina AC. Independent predictors of isolated clinic ('white-coat') hypertension. J Hypertens 2001; 19:1015-1020.

130 Verdecchia P, Schillaci G, Borgioni C, Ciucci A, Zampi I, Gattobigio R, et al. White-coat hypertension and white-coat effect: similarities and differences. Am J Hypertens 1995; 8:790-798.

131 Myers MG, Reeves RA. White-coat phenomenon in patients receiving antihypertensive therapy. Am J Hypertens 1991; 4:844-849.

132 Myers MG, Reeves RA. White-coat effect in treated hypertensive patients: sex difference. J Hum Hypertens 1995; 9:729-733.

133 Wing LMH, Brown MA, Beilin L, Ryan P, Reid CM, on behalf of the ANBP2 Management Committee and Investigators. 'Reverse white coat hypertension' in older hypertensives. J Hypertens 2002; 20:639-644.

134 Pickering T, Davidson K, Gerin W, Schwartz JE. Masked hypertension. Hypertension 2002; 40:795-796.

135 Asmar RG, Girerd XJ, Brahimi M, Safavian A, Safar ME. Ambulatory blood pressure measurement, smoking and abnormalities of glucose and lipid metabolism in essential hypertension. J Hypertens 1992; 10:181-187.

136 Staessen JA, Fagard R, Thijs L, Celis H, Arabidze GG, Birkenhager $\mathrm{WH}$, et al. Randomised double-blind comparison of placebo and active treatment for older patients with isolated systolic hypertension. Lancet 1997; 350:757-764

137 Verdecchia P, Schillaci G, Guerrieri M, Gatteschi C, Benemio G, Boldrini $F$, et al. Diurnal blood pressure changes and left ventricular hypertrophy in essential hypertension. Circulation 1990; 81:528-536.

138 Verdecchia P, Schillaci G, Borgioni C, Ciucci A, Gattobigio R, Porcellat 
C. Nocturnal pressure is the true pressure. Blood Press Monit 1996; 1: S81-S85.

139 Okhubo T, Hozawa A, Yamaguchi J, Kikuya M, Ohmori K, Michimata M, et al. Prognostic significance of the nocturnal decline in blood pressure in individuals with and without high 24 -h blood pressure: the Ohasama study. J Hypertens 2002; 20:2183-2189.

140 Okhubo T, Imai Y, Tsuji I, Nagai K, Watanabe N, Minami N, et al. Relation between nocturnal decline in blood pressure and mortality. The Ohasama study. Am J Hypertens 1997; 10:1201-1207.

141 Bellomo G, Narducci PL, Rondoni F, Pastorelli G, Stangoni G, Angeli G, Verdecchia P. Prognostic value of 24 -hour blood pressure in pregnancy. JAMA 1999; 282:1447-1452.

142 Halligan A, O'Brien E, O'Malley K, Mee F, Atkins N, Conroy R, et al. Twenty-four hour ABPM in a primigravid population. J Hypertens 1993; 11:869-873.

143 Hermida RC, Ayala DE. Diagnosing gestational hypertension and preeclampsia with the 24-hour mean of blood pressure. Hypertension 1997; 30:1531-1537.

144 Higgins JR, Walshe JJ, Halligan A, O'Brien E, Conroy R, Darling MR. Can 24 hour ABPM predict the development of hypertension in primigravidae? Br J Obstet Gynaecol 1997; 104:356-362.

145 Halligan AWF, Shennan A, Lambert PC, Taylor DJ, de Swiet M. Automated blood pressure measurement as a predictor of proteinuric pre-eclampsia. Br J Obstet Gynaecol 1997; 104:559-562.

146 Penny JA, Halligan AWF, Shennan AH, Lambert PC, Jones DR, de Swiet $\mathrm{M}$, et al. Automated, ambulatory, or conventional blood pressure measurement in pregnancy: which is the better predictor of severe hypertension? Am J Obstet Gynecol 1998; 178:521-526.

147 Churchill D, Perry IJ, Beevers DG. ABPM in pregnancy and fetal growth. Lancet 1997; 349:7-10.

148 Sturrock ND, George E, Pound N, Stevenson J, Peck GM, Sowter H. Non-dipping diurnal blood pressure and renal impairment are associated with increased mortality in diabetes mellitus. Diabet Med 2000; 17:360-364.

149 Lurbe E, Redon J, Kesani A, Pascual JM, Tacons J, Alvarez V, et al. Increase in nocturnal blood pressure and progression to microalbuminuria in type 1 diabetes. N Engl J Med 2002; 347:797-805.

150 Strachan MW, Gough K, McKnight JA, Padfield PL. Ambulatory blood pressure monitoring: is it necessary for the routine assessment of hypertension in people with diabetes? Diabet Med 2002; 19:787-789.

151 Owens P, O'Brien ET. Hypotension in patients with coronary disease can profound hypotensive events cause myocardial ischaemic events? Heart 1999; 82:477-481.

152 Mallion J-M, Baguet J-P, Siché J-P, Tremel F, De Gaudemaris R. Clinical value of ABPM monitoring. J Hypertens 1999; 17:585-595

153 White W. Guidelines on the clinical utility of ABPM. Blood Press Monit 1998; 3:181-184.

154 Asmar $\mathrm{R}$, Lacourcière $\mathrm{Y}$, on behalf of the Candesartan Cilexetil in Hypertension Ambulatory Measurement of Blood Pressure (CHAMP) Study Investigators. A new approach to assessing antihypertensive therapy: effect of treatment on pulse pressure. J Hypertens 2000; 18:1683-1690.

155 Parati G, Ravogli A, Mutti E, Santucciu C, Omboni S, Mancia G. Ambulatory blood pressure monitoring in the evaluation of antihypertensive drugs. J Hypertens 1994; 12 (suppl 8):S9-S15.

156 Omboni S, Parati G, Zanchetti A, Mancia G. Calculation of trough-to peak ratio of antihypertensive treatment from ambulatory blood pressure: methodological aspects. J Hypertens 1995; 13:1105-1112.

157 Parati G, Omboni S, Rizzoni D, Agabiti-Rosei E, Mancia G. The smoothness index: a new reproducible and clinically relevant measure of the homogeneity of the blood pressure reduction by treatment in hypertension. J Hypertens 1998; 16:1685-1691.

158 Palatini PP, Dorigatti F, Roman E, Gioinazzo P, Piccolo D, De Venuto G, et al. White-coat hypertension. A selection bias? J Hypertens 1998; 16:977-984.

159 O'Brien E, Beevers G, Lip GHY. ABC of hypertension. Part IV Automated sphygmomanometry: self blood pressure measurement. BMJ 2001; 322:1167-1170.

160 Laher M, O'Boyle C, Kelly J, O'Brien E, O'Malley K. Home measurement of blood pressure: training of relatives. Ir Med J 1981; 74:113-114.

161 Asmar R, Zanchetti A, on behalf of the Organizing Committee and participants. Guidelines for the use of self-blood pressure monitoring: a summary report of the first international consensus conference. J Hypertens 2000; 18:493-508.

162 Asmar R. Proceedings from the First International Consensus Conference on Self-Blood Pressure Measurement. Blood Press Monit 2000; 5:91-92.

163 O'Brien E, De Gaudemaris R, Bobrie G, Agabiti Rosei E, Vaisse B, and the participants of the First International Consensus Conference on Blood Pressure Self-Measurement. Devices and validation. Blood Press Monit 2000; 5:93-100.

164 Staessen J, Thijs L, and the participants of the First International Consensus Conference on Blood Pressure Self-Measurement. Development of diagnostic thresholds for automated self-measurement of blood pressure in adults. Blood Press Monit 2000; 5:101-109.

165 Mengden T, Chamontin B, Phong Chau NG, Gamiz JLP, Chanudet X, and the participants of the First International Consensus Conference on Blood Pressure Self-Measurement. User procedure for self-measurement of blood pressure. Blood Press Monit 2000; 5:111-129.

166 Herpin D, Pickering T, Stergiou G, de Leeuw P, Germano G, and the participants of the First International Consensus Conference on Blood Pressure Self-Measurement. Clinical applications and diagnosis. Blood Press Monit 2000; 5:131-135.

167 Imai Y, Poncelet P, DeBuyzere M, Padfield PL, Van Montfrans GA, and the participants of the First International Consensus Conference on Blood Pressure Self-Measurement. Prognostic significance of self-measurements of blood pressure. Blood Press Monit 2000; 5:137-143.

168 Denolle T, Waeber B, Kjeldsen S, Parati G, Wilson M, Asmar R, and the participants of the First International Consensus Conference on Blood Pressure Self-Measurement. Self-measurement of blood pressure in clinical trials and therapeutic applications. Blood Press Monit 2000; 5:145-149.

169 Parati G, Asmar R, Stergiou G. Self blood pressure monitoring at home by wrist devices: a reliable approach? J Hypertens 2002; 20:573-578.

170 Grassi G, Foglia G, Dell'Oro R, Seravalle G, Boari S, Mancia G. Reproducibility of home blood pressure monitoring by a new oscillometric wrist device. J Hypertens 2001; 19 (suppl 2):22-23.

171 Uen E, Weisser B, Wieneke P, Mengden TL. Evaluation of the performance of a wrist blood pressure measuring device with a position sensor compared to ambulatory 24-hour blood pressure measurements. Am J Hypertens 2002; 9:787-792.

172 Imai Y, Nishiyama A, Sekino M, Aihara A, Kikuya M, Ohkubo T, et al. Characteristics of blood pressure measured at home in the morning and in the evening; the Ohasama study. J Hypertens 1999; 101: 889-898.

173 Stergiou GS, Skeva II, Zourbaki AS, Mountokalakis TD. Self-monitoring of blood pressure at home: how many measurements are needed? J Hypertens 1998; 16:725-731.

174 Mengden T, Hernandez Medina RM, Beltran B, Alvarez E, Kraft K, Vetter $\mathrm{H}$. Reliability of reporting self-measured blood pressure values by hypertensives patients. Am J Hypertens 1998; 11:1413-1417.

175 Grim CM, Grim CE. A curriculum for the training and certification of blood pressure measurement for health care providers. Can J Cardiol 1995; 11 (suppl H):38H-42H.

176 The Cardiovascular Institute. Hypertension and self-blood pressure measurement [CD-ROM] 2002. Available from The Cardiovascular Institute, The Cardiovascular Institute, 21 bd Delessert, 75016, Paris, France.

177 Imai Y, Munakata M, Tsuji I, Ohkubo T, Yoshino H, Watanabe N, et al. Seasonal variation in blood pressure in normotensive women studied by home measurements. Clin Sci 1996; 95:55-60.

178 Tsuji I, Imai Y, Nagai K, Ohkubo T, Watanabe N, Minami N, et al. Proposal of reference values for home blood pressure measurement. Prognostic criteria based on a prospective observation of the general population in Ohasama, Japan. Am J Hypertens 1997; 10:409-418.

179 Ohkubo T, Imai Y, Tsuji I, Nagai K, Sakuma M, Watanabe N, et al. Home blood pressure measurement has a stronger predictive power for mortality than does screening blood pressure measurement: a population-based observation in Ohsama, Japan. J Hypertens 1998; 16:971-975.

180 Thijs L, Staessen J, Celis H, De Gaudemaris R, Imai Y, Julius S, et al. Reference values for self-recorded blood pressure. A meta-analysis of summary data. Arch Intern Med 1998; 158:481-488.

181 Appel LJ, Stason WB. Ambulatory blood pressure monitoring and blood pressure self-measurement in the diagnosis and management of hypertension. Ann Intern Med 1993; 118:867-882.

182 Brueren MM, Schouten HJ, de Leeuw PW, van Montfrans GA, van Ree JW. A series of self-measurements by the patient is a reliable alternative to ambulatory blood pressure measurement. $\mathrm{Br} J$ Gen Pract 1998 ; 48:1585-1589.

183 Stergiou GS, Zourbaki AS, Skeva II, Mountokalakis TD. White coat effect detected using self-monitoring of blood pressure at home: comparison with ambulatory blood pressure. Am J Hypertens 1998 11:820-827.

184 Sega R, Trocino G, Lanzarotti A, Carugo S, Cesana G, Schiavina R, et al. Alterations in cardiac structure in patients with isolated office, 
ambulatory or home hypertension. Data from the PAMELA study. Circulation 2001; 104:1385-1392.

185 Parati G, Ulian L, Sampieri L, Palatini P, Villani A, Vanasia A, on behalf of the Study on Ambulatory Monitoring of Blood Pressure and Lisinopril Evaluation (SAMPLE) Study Group. Attenuation of the 'white-coat effect' by antihypertensive treatment and regression of target organ damage. Hypertension 2000; 35:614-620.

186 Imai Y, Ohkubo T, Hozawa A, Tsuji I, Matsubara M, Araki T, et al. Usefulness of home blood pressure measurements in assessing the effect of treatment in a single-blind placebo-controlled trial. $J$ Hypertens $2001 ; 19: 179-185$.

187 Ménard J, Chatellier G, Day M, Vaur L. Self-measurement of blood pressure at home to evaluate drug effects by the trough:peak ratio. $J$ Hypertens 1994; 12 (suppl 8):S21-S25.

188 Edmonds D, Foerster EG, Greminger P, Siegenthaler W, Vetter W. Does self-measurement of blood pressure improve patient compliance in hypertension? J Hypertens 1985; 3 (suppl 1):31-34.

189 Friedman RH, Kazis LE, Jette A, Smith MB, Stollerman J, Torgerson J, et al. A telecommunications system for monitoring and counseling patients with hypertension. Impact on medication adherence and blood pressure control. Am J Hypertens 1996; 9:285-292.

190 Ibrahim MM, Tarazi RC, Dustan HP, Gifford RW. Electrocardiogram in evaluation of resistance to antihypertensive therapy. Arch Intern Med $1977 ; 137: 1125-1129$

191 Verdecchia P, Bentivoglio M, Providenza M, Savino K, Corea L. Reliability of home self-recorded arterial pressure in essential hypertension in relation to the stage of the disease. In: Germano G, (editor): Blood pressure recording in the clinical management of hypertension. Rome: Ediziono Pozzi; 1985. pp. 40-42.

\section{Appendix \\ European Society of Hypertension Working Group on Blood Pressure Monitoring}

ROLAND ASMAR, The Cardiovascular Institute, 21 bd Delessert, 75016, Paris, France.

LAWRIE BEILIN, Department of Medicine, University of Western Australia, GPO Box X2213, Royal Perth Hospital, Perth, West Australia 6847, Australia.

Denis L. Clement, Afdeling Hart-en Vaatziekten, Universitair Ziekenhuis, De Pintelaan 185, B-9000 Gent, Belgium.

PeTER DE LeEuW, Interne Geneeskunde, Academisch Ziekenhuis, P. Debyelaan 25, Postbus 5800, 6202 AZ Maastricht, The Netherlands.

ROBERT FAGARD, Katholieke Universiteit Leuven, Hypertensie en Cardiovasculaire Inevalidatie Eenheid, Inwendige Geneeskunde-Cardiologie, UZ Gasthuisberg, Herestraat 49, 3000 Leuven, Belgium.

YUTAKA IMAI, Department of Clinical Pharmacology and Therapeutics, Tohoku University Graduate School of Pharmaceutical Science and Medicine, 1-1 Seiryocho, Aoba-ku, Sendai 980-8574, Japan.

Jean-Michel Mallion, Médecine Interne et Cardiologie, Chef de Service, Centre Hospitalier Universitaire de Grenoble, BP 217-38043 Grenoble Cedex, France.

Giuseppe Mancia, Universita Degli Studi di Milano-
Bicocca, Cattedra di Medicina Interna, Ospedale San Gerardo Dei Tintori, Via Donizetti 106, 20052 Monza, Italy.

Thomas Mengden, University Clinic Bonn, Department of Internal Medicine, Wilhelmstrasse 35, 53111 Bonn, Germany.

Martin G. Myers, Division of Cardiology, Sunnybrook and Women's College Health Sciences Centre, 2075 Bayview Avenue, Toronto, Ontario M4N 3M5, Canada.

EOIN O'BRIEN (Chairman), Blood Pressure Unit and Royal College of Surgeons in Ireland Medical School, Beaumont Hospital, Dublin 9, Ireland.

Paul Padfield, Department of Medicine, Western General Hospital, Edinburgh EH4 2XU, UK.

Paolo Palatini, Dipartimento di Medicina Clinica e Sperimentale, Universita' di Padova, Via Giustiniani 2, I-35128 Padua, Italy.

Gianfranco Parati, Department of Clinical Medicine, Prevention and Applied Biotechnologies, University of Milano-Bicocca and Ospedale San Luca, IRCCS, Istituto Auxologico Italiano, Via Spagnoletto 3, 20149 Milan, Italy.

Thomas G. Pickering, Director, Integrative and Behavioral Cardiovascular Health Program, Mount Sinai Medical Center, New York, NY 10029-6574, USA.

Josep Redon, Hypertension Clinic, Internal Medicine, Hospital Clinico, University of Valencia, Avda Blasco Ibañez 17, 46010 Valencia, Spain.

Jan Staessen, Katholieke Universiteit Leuven, Hypertensie en Cardiovasculaire Revalidatie Eenheid, Inwendige Geneeskunde-Cardiologie, UZ Gasthuisberg, Herestraat 49, 3000 Leuven, Belgium.

George Stergiou, Hypertension Center, Third University Department of Medicine, Sotiria Hospital, Athens, Greece.

Gert van MontFrans, Academisch Medisch Centrum, Interne Ziekten, Meibergdreef 9, AZ 1005 Amsterdam, The Netherlands.

Paolo Verdecchia, Departimento di Malattie Cardiovascolari, Ospedale R. Silvestrini, Perugia, Italy.

Bernard WAEber (Secretary), Centre Hospitalier Universitaire Vaudois, Division d'Hypertension, Départe- 
ment de Médecine Interne, 1011 Lausanne, Switzerland.

WiLliam White, Section of Hypertension and Vascular Diseases, The University of Connecticut Health Center, 263 Farmington Avenue, Farmington, Connecticut 06030-3940, USA. 